\title{
LES ISOSPORA DU MOINEAU
}

\section{II - Études sur la biologie}

\author{
O. GRULET* ${ }^{*}$ I. LANDAU**, P. MILLET** et D. BACCAM**
}

RÉSUMÉ. L'allure générale des infections, la morphologie et l'évolution des stades tissulaires des Isospora du Moineau sont étudiées.

L'ensemble des observations, effectuées chez des Moineaux capturés à différentes périodes de l'année et autopsiés à différentes heures de la journée, révèle :

1) que l'évolution du parasitisme est déterminée par des facteurs climatiques ou nycthéméraux. Parmi les 18 espèces observées, 7 sont présentes toute l'année, et 11 seulement épisodiquement (6 d'entre elles sont très rares). La période de transmission paraît être l'été : c'est pendant les mois chauds de l'année que les infestations sont les plus fortes et que sont observés les stades de multiplication particuliers faisant suite à l'ingestion des oocystes. Au cours du nycthémère on assiste à une succession de stades tissulaires qui, pour toutes les espèces, aboutit à l'excrétion des oocystes pendant l'après-midi ;

2) que les formes tissulaires présentent une grande diversité dans leur morphologie, leur évolution et leur localisation (épithélium des villosités, glandes de Lieberkühn, système réticulo-endothélial), et que ce polymorphisme correspond à une pluralité des cycles biologiques.

Les stades tissulaires de chaque espèce n'ont pu être déterminés en raison du polyparasitisme; cependant il a été possible de rattacher un grand nombre d'entre eux à 3 groupes d'espèces :

a) Espèces à cycle court : l'évolution serait limitée à l'épithélium des villosités. La schizogonie gamétogène, la gamétogonie et la sporogonie siègent dans l'épithélium des villosités intestinales et suivent un rythme nycthéméral : schizogonie apparaissant à la tombée de la nuit et ne durant que quelques heures, suivie successivement par la gamétogonie, la sporogonie et l'excrétion des oocystes qui se termine le lendemain au moment de l'apparition, à la tombée de la nuit, d'une nouvelle schizogonie gamétogène.

b) Espèces présentes toute l'année et ayant donc un cycle prolongé. La schizogonie gamétogène, la gamétogonie et la sporogonie siègent dans l'épithélium des villosités et ont le même rythme nycthéméral que dans le groupe précédent. L'entretien du parasitisme des villosités est assuré chaque jour pendant toute l'année par des stades évoluant dans d'autres tissus :

- dans les glandes de Lieberkühn pour un premier groupe d'espèces. Il s'agit d'une schizogonie subintrante, qui évolue à bas bruit pendant le jour et devient abondante pendant la nuit ;

- dans le système réticulo-endothélial pour un deuxième groupe d'espèces. Il s'agit de trophozoïtes d'attente apparus pendant la période de transmission, qui réensemenceraient les villosités le soir.

- Laboratoire d'Helminthologie et de Parasitologie comparée, École Pratique des Hautes Études, 61, rue Buffon, F 75231 Paris Cedex 05.

** Laboratoire de Zoologie (Vers), associé au C.N.R.S., Muséum National d'Histoire Naturelle, 61, rue Buffon, F 75231 Paris Cedex 05.

I. Cette étude a reçu l'appui financier du Programme spécial P.N.U.D./Banque mondiale/

O.M.S. de Recherche et de Formation concernant les maladies tropicales.

Accepté le 20 mars 1985. 
Il est suggéré que les modes de division endomérogonique et ectomérogonique correspondent à 2 processus biologiques distincts : - l'un à l'origine de la gamétogonie, - l'autre à l'origine des formes d'attente.

Il est vraisemblable que le cycle intestinal du Toxoplasme qui figure actuellement dans tous les traités est erroné. Il n'y aurait pas, comme cela est admis, succession de divisions tantôt ecto-, tantôt endomérogoniques, mais deux lignées bien distinctes.

\section{Isospora of sparrows. II — Studies on biology.}

SUMMARY. The general evolution of infections, the morphology and development of tissue stages of Isospora of sparrows are studied.

Observations made on sparrows captured at various times of the year and autopsied at diflerent hours of the day show that :

1) evolution of parasitism is determined by climatic or circadian factors. Of 18 species found, 7 are present all throughout the year and 11 are only episodic ( 6 are very rare). The transmission period appears to be summer : infections are the heaviest during the warm months of the year and the special division stages following the ingestion of oocysts can then be found. During the circadian period, there is a succession of tissue stages that, for all species, ends up by the excretion of oocysts during the afternoon ;

2) tissue stages present a vast diversity in morphology, evolution and localisation (epithelium of villi, crypts of Lieberkühn, reticulo-endothelial system); this polymorphism is related to differences in life cycles.

Because of the polyparasitism it was not possible to correlate the various tissue stages to the corresponding species but only to 3 groups of species :

a) Species which have a short cycle : their development is very likely confined to the villi's epithelium. Gametogenetic schizogony, gametogony, sporogony are located in the epithelium of the intestinal villi and follow a circadian rythm : schizogony starts at the beginning of night and lasts only a few hours, followed successively by gametogony, sporogony and excretion of oocysts which ends the following night when a new schizogony begins.

b) Species which are present all the year and have a prolonged life cycle. Ganetogonic schizogony, gametogony, and sporogony are located in the villi's epithelium and follow the same circadian rythm as in the previous group. Parasitism in the intestinal villi appears to be initiated every night during the whole year by stages developing in other tissues :

- the crypts of Lieberkühn in a first group of species : a schizogony evolves at a low level during the day and becomes abundant at night ;

- the reticulo-endothelial system in a second group of species : latent trophozoites appear during the transmission period and initiate schizogony in the villi at night.

It is suggested that the mode of division, ectomerogony and endomerogony, corresponds to two distinct biological processes : - one originating gametogony, - the other, latent stages.

The intestinal cycle of Toxoplasma, as classically presented, is likely to be erroneous ; endomerogonic and ectomerogonic divisions which admittedly succeed each other should be considered as belonging to two distinct lines.

Notre travail porte sur un grand nombre de Moineaux capturés à différentes périodes de l'année, autopsiés dès leur capture ou après un séjour, de durée variable, dans l'animalerie.

Le matériel dont nous disposons, comprenant toujours un polyparasitisme important, l'étude biologique de chaque espèce ne peut porter que sur les seuls stades 
identifiables avec certitude : les oocystes mûrs. Elle révèle que la présence et le niveau du parasitisme de 7 espèces varient peu au cours de l'année, alors que l'infection par les 11 autres espèces (5 espèces décrites et 6 non décrites) est irrégulière et discontinue.

Pour les stades tissulaires que nous ne pouvons pas identifier de façon spécifique, cette étude montre :

- que les stades sexués (gamétocytes) et les oocystes immatures intracellulaires varient peu dans leur siège (épithélium des villosités), leurs caractères morphologiques et leur évolution ;

- que les stades asexués, par contre, présentent une très grande diversité dans leur localisation (épithélium des villosités, système réticulo-endothélial de tous les tissus et épithélium des cryptes de Lieberkühn), leur morphologie et leurs caractères évolutifs (certains se multiplient activement, d'autres sont en attente dans les tissus).

Il nous est apparu que la nature de ces stades asexués ne peut être interprétée que si l'on admet l'existence de deux types de cycles :

- un cycle court, aigu, guérissant rapidement et limité à l'épithélium des villosités, de même type que celui d'I. canaria Box, 1977 (cf. plus loin). Les espèces, déterminant une infection irrégulière et discontinue, ont un cycle de ce type ;

- un cycle prolongé, comprenant des stades asexués et sexués dans l'épithélium des villosités et une grande variété de stades asexués évoluant tout au long de l'année dans d'autres tissus ; ces derniers entretiendraient le parasitisme des villosités. Les espèces ayant ce type de cycle sont présentes tout au long de l'année et se répartissent en deux groupes : le premier présentant un tropisme pour le système réticulo-endothélial de tous les organes, le second pour l'épithélium des glandes de Lieberkühn.

Dans un premier chapitre, nous rappelons brièvement les données de la littérature sur les Isospora. Dans un deuxième chapitre, les variations du parasitisme par les différentes espèces sont étudiées. Enfin, dans un troisième chapitre, nous décrivons les formes tissulaires rencontrées chez les Moineaux et proposons une interprétation des cycles.

\section{Matériel et méthodes}

\section{a - Origine du matériel}

Les Moineaux utilisés dans ce travail ont été capturés à l'aide de filets japonais dans les localités suivantes :

- en France:

- "La Bunelière ", Beaulieu (Orne) ; 100 Moineaux (de novembre 1979 à avril 1983).

- Bignon-Mirabeau (Loiret) ; 86 Moineaux (d'avril 1982 à avril 1983).

- Clères (Seine-Maritime) ; 7 Moineaux (avril 1981). 
- Paris ; 32 Moineaux (juillet 1980, février 1982, octobre 1982).

- Jouy-en-Josas (Yvelines) ; 27 Moineaux (mars 1982, mai 1982).

- en Australie:

- Adélaïde ; 4 Moineaux (novembre 1981).

\section{b - Méthodes d'étude}

1) Pour l'étude de l'évolution du parasitisme au cours des 24 heures

Trois lots de Moineaux sont autopsiés, dès leur capture, à différents intervalles de temps.

Le décalage d'une heure, lié à l'horaire d'été suivi en Europe occidentale, est supprimé. En été, comme en hiver, les heures indiquées dans le texte ont donc uniformément un retard de soixante minutes sur l'heure solaire.

- en avril 1980:8 Moineaux (428 CL à $435 \mathrm{CL}$ ) sacrifiés à 14 h, 16 h, 18 h, 20 h, $22 \mathrm{~h}, 3 \mathrm{~h}, 5 \mathrm{~h}, 7 \mathrm{~h}$;

— en novembre 1980: 12 Moineaux (700 XV à $711 \mathrm{XV}$ ) sacrifiés à $13 \mathrm{~h}, 15 \mathrm{~h}$,

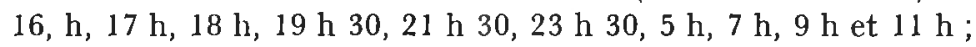

- en mai 1981 : 11 Moineaux (39 LU, $46 \mathrm{LU}, 48 \mathrm{LU}, 55 \mathrm{LU}, 62 \mathrm{LU}, 69 \mathrm{LU}$,

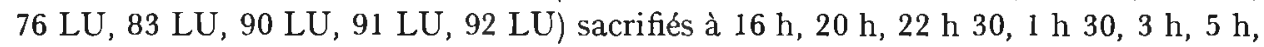
$7 \mathrm{~h}, 9 \mathrm{~h} \mathrm{30,12}$ h, 12 h 30 et 12 h 45.

Un 4 e lot de 5 Moineaux (131 XV, $132 \mathrm{XV}, 137 \mathrm{XV}, 139 \mathrm{XV}, 140 \mathrm{XV})$, de juillet 1980, est sacrifié à 11 h 30,13 h 30,17 h 30,19 h 30 et 22 h 30,18 jours après la capture.

2) Pour l'étude in vitro

L'excystation des sporozoïtes a été obtenue selon la technique de Jackson, 1964.

Les cultures primaires de cellules hépatiques de Canari sont obtenues par perfusion enzymatique du foie par la collagénase (type IV de Sigma) selon les techniques décrites par Lambiotte et coll., 1981 pour le Rat. Les cultures sont effectuées dans des boîtes de Pétri de $3,5 \mathrm{~cm}$ de diamètre. Le milieu est du MEM additionné de $10 \%$ de sérum de veau fotal, et de 200 UI de pénicilline et $200 \mu \mathrm{g}$ de streptomycine par millilitre.

L'étalement des cellules de Canari est plus lent que celui des cellules de Rongeur (30 à 48 heures, au lieu de moins de 4 heures). On obtient une culture d'hépatocytes et de fibroblastes.

L'infestation des cultures est effectuée plus de 72 heures après la perfusion.

\section{c - Conditions de captivité}

Les Moineaux sont gardés dans l'animalerie éclairée par la lumière du jour, à une température moyenne de $25^{\circ} \mathrm{C}$. Ils sont placés dans des cages individuelles, et l'eau et les graines sont changées tous les deux jours ou tous les jours. Il n'a pas été possible de les isoler pour éviter les recontaminations. 


\section{I - Rappel des données de la littérature}

\section{A - Cycle classique d'une Coccidie intestinale}

Nous prendrons comme exemple celui d'I. canaria décrit par Box en 1977, parasite de Serinus canarius dont le schéma est détaillé plus loin (fig. 1).

Il s'agit d'un cycle monoxène, la contamination se faisant par ingestion d'oocystes disséminés dans le milieu extérieur.

Il comprend une schizogonie, une gamétogonie et une sporogonie siégeant uniquement dans l'épithélium des villosités.

L'infection est limitée dans le temps et la guérison survient à brève échéance.

Dans ce cycle, toutes les divisions schizogoniques se font par ectomérogonie, c'est-à-dire que les mérozoïtes se forment par bourgeonnement à la périphérie du schizonte ; à maturité il persiste fréquemment un corps résiduel cytoplasmique bien limité.

Dans ses grandes lignes, l'évolution de la gamétogonie et de la sporogonie est très comparable chez toutes les espèces d'Isospora.

La période prépatente est le laps de temps entre l'ingestion de l'oocyste contaminant et l'excrétion des premiers oocystes ; la période patente est le laps de temps entre le début de l'excrétion des oocystes et la guérison. Pendant cette période, les schizogonies intestinales persistent et entretiennent la gamétogonie et la sporogonie.

Dans la réalité, le schéma classique monoxène décrit plus haut est rarement observé; la plupart des Coccidies sont hétéroxènes (Landau, 1973) ; leur cycle comprend une phase aiguë asexuée et sexuée dans l'épithélium intestinal, et des stades d'attente dans d'autres tissus; ceux-ci ne poursuivent leur évolution que chez un deuxième hôte prédateur qui les ingère ; c'est le cas de Toxoplasma (Hutchison et Dunachie, 1971), des Sarcocystis (Fayer, 1974), d'Isospora felis (Dubey et Frenkel, 1972), et des Eimeria de Poissons (Landau, 1975 et 1982, Solangi et Overstreet, 1980).

\section{B - Données de la littérature sur les Isospora de Passériformes}

Les connaissances sur la biologie des Isospora de Passériformes sont relativement fragmentaires.

1) Périodicité : Boughton (1933 et 1937) a, le premier, découvert la périodicité journalière de l'excrétion des oocystes et en a déterminé l'horaire. Chez le Moineau, il montre que : dans des conditions ordinaires, les Oiseaux excrètent des oocystes entre $13 \mathrm{~h}$ et $20 \mathrm{~h}$; en inversant les périodes d'éclairages diurne et nocturne, il inverse également le rythme d'excrétion des oocystes.

2) Durée du parasitisme: Boughton (1937) note que des Moineaux isolés dans leurs cages, dans des conditions rendant toute recontamination par des oocystes impossible, conservent le même niveau de parasitisme pendant les 2 mois de l'expérience. Il suggère que l'infection acquise dans le nid se prolonge pendant toute la vie du Moineau. 
Box (1977) étudie expérimentalement 2 Isospora du Canari : elle montre que chez $I$. canaria l'excrétion des oocystes est limitée à deux semaines, alors que chez I. serini l'excrétion des oocystes persiste plus de 4 mois.

3) Recontaminations: Boughton (1937) note que l'ingestion d'oocystes par des Moineaux déjà infectés augmente de façon notable l'intensité du parasitisme.

4) Localisation des stades tissulaires : Box (1977), en étudiant expérimentalement les deux espèces d'Isospora du Canari, $I$. serini et $I$. canaria, montre que leur évolution s'effectue dans deux localisations bien différentes : I. canaria se développe exclusivement dans l'épithélium intestinal, $I$. serini évolue d'abord dans les cellules réticulo-endothéliales et ensuite intestinales.

Desser (1980) décrit chez Hesperiphona vespertina et Pheneticus ludovicianus (Fringillidae) une Isospora proche d'I. serini : la multiplication dans les mononucléaires du système réticulo-endothélial, au printemps, est très importante, provoquant une forte mortalité chez les Oiseaux.

Stabler et Kitzmiller (1972) décrivent les stades tissulaires d'I. petrochelidon chez des oisillons d'Hirondelles contaminés depuis 5 jours avec des oocystes; la gamétogonie et la sporogonie siègent dans l'épithélium des villosités; des schizontes sont décrits dans les glandes de Lieberkühn.

5) Évolution, siège et horaires de la sporogonie: En 1982, Grulet et coll. étudient des infections naturelles polyparasitaires chez des Moineaux sacrifiés à différentes heures de la journée. Ils observent que : chaque espèce est localisée à un niveau particulier le long de l'intestin et que les oocystes et gamétocytes sont groupés en populations homogènes dans les villosités; chaque espèce suit un horaire très précis ; la majorité des oocystes est excrétée pendant environ 4 heures; il existe fréquemment un décalage pouvant atteindre $4 \mathrm{~h}$ au maximum entre les différentes espèces.

6) Stades de multiplication: En utilisant la classification de Hammond (1973), Box (1977) décrit, au cours de la période prépatente d'I. serini, 2 types de schizontes, les uns ectomérogoniques, les autres endomérogoniques.

7) Cultures des stades tissulaires: Box (1970) obtient l'évolution de trois générations schizogoniques d'Isospora de Moineaux capturés à Galveston (Texas) dans des cellules d'embryon de Poulet et de Canari.

Millet (1984) obtient, avec des Isospora de Moineaux de France, deux types d'évolution dans des cultures primaires d'hépatocytes de Canari ( $P l . I V)$ :

a) la première, attribuée aux espèces à tropisme réticulo-endothélial : division du sporozoïte en 2 moitiés, chacune contenant un cristalloïde et un noyau ; première génération schizogonique à partir d'un demi-sporozoïte; nombreuses générations schizogoniques successives.

b) la seconde, attribuée aux espèces localisées uniquement dans l'épithélium intestinal : transformation du sporozoïte directement en schizonte sans division préalable. 


\section{IJ - Étude des espèces : oocystes présents aux différentes périodes de l'année}

Nous rectifions et complétons ici (tableau I) les observations précédentes (Grulet et coll., 1982) sur les espèces observées aux différentes périodes de l'année. Le tableau I diffère de celui précédemment publié, car seuls les résultats concernant des Moineaux étudiés moins de 5 jours après leur capture sont conservés. Par ailleurs, nous n'étions pas sûrs qu'I. miltgeni ait été absente lors de nos premières observations ou seulement identifiée tardivement en novembre 1980, date à laquelle nous l'avons observée pour la première fois. Des observations faites depuis permettent d'affirmer que cette espèce est rare.

Les espèces suivantes sont présentes toute l'année : I. boxae, I. jacobfrenkeli, I. gonnetae, I. iansmithi, I. fatiguei, I. michaelbakeri, I. mikei.

I. yesi, I. spratti, I. nancyae, I. miltgeni, I. kouyatei n'apparaissent qu'à certaines périodes de l'année. I. yesi a été observé de juillet à novembre, $I$. konyatei en avril et juin, I. spratti, I. nancyae et $I$. miltgeni de novembre à juin.

TABLEAU I. - Espèces d'Isospora du moineau présentes de juillet 1980 à juin 1981 à

"La Bunelière ", Beaulieu (Orne) ; Moineaux étudiés moins de 5 jours après leur capture.

Les chiffres indiquent le nombre d'Oiseaux positifs.

\begin{tabular}{|c|c|c|c|c|c|c|c|c|c|}
\hline & $\begin{array}{l}\text { Juillet } \\
1980\end{array}$ & $\begin{array}{l}\text { Août } \\
1980\end{array}$ & $\begin{array}{l}\text { Sept. } \\
1980\end{array}$ & $\begin{array}{c}\text { Octobre } \\
1980\end{array}$ & $\begin{array}{l}\text { Nov. } \\
1980\end{array}$ & $\begin{array}{c}\text { Janvier } \\
1981\end{array}$ & $\begin{array}{c}\text { Février } \\
1981\end{array}$ & $\begin{array}{l}\text { Avril } \\
1981\end{array}$ & $\begin{array}{l}\text { Juin } \\
1981\end{array}$ \\
\hline I. boxae & 5 & 3 & 3 & l & 7 & 3 & 6 & 7 & 13 \\
\hline I. jacobfrenkeli & 2 & 2 & 1 & 0 & 5 & l & 2 & 5 & 11 \\
\hline I. gonnetae & 4 & 3 & 2 & 1 & 4 & 1 & 5 & 2 & 7 \\
\hline I. iansmithi & 3 & 2 & 2 & 0 & 6 & 1 & 5 & 5 & 11 \\
\hline I. fatiguei & 3 & 1 & 2 & 1 & 5 & 2 & 6 & 2 & 11 \\
\hline I. kouyatei & 0 & 0 & 0 & 0 & 0 & 0 & 0 & 1 & 1 \\
\hline I. michaelbakeri & 3 & 2 & 3 & 1 & 2 & 1 & 5 & 7 & 11 \\
\hline I. miltgeni & 0 & 0 & 0 & 0 & 0 & 2 & 3 & 0 & 5 \\
\hline I. nancyae & 0 & 0 & 0 & 0 & 2 & I & 5 & 0 & 2 \\
\hline I. mikei & 5 & 1 & 3 & 1 & 2 & 1 & 3 & 3 & 6 \\
\hline I. spratti & 0 & 0 & 0 & 0 & 2 & 0 & 1 & 2 & 3 \\
\hline I. yesi & 1 & 1 & 1 & 0 & 1 & 0 & 0 & 0 & 0 \\
\hline $\begin{array}{l}\text { Nombre total } \\
\text { de Moineaux } \\
\text { examinés }\end{array}$ & 5 & 3 & 3 & 1 & 7 & 3 & 6 & 7 & 13 \\
\hline
\end{tabular}

\section{III - Étude des cycles}

\section{A - Gamétogonie et sporogonie intra-cellulaires}

1) Allure générale des infections

- au cours de l'année : on note des variations dans la richesse des infections (tableau II) : c'est en été que les gamétocytes et oocystes sont les plus abondants. 
TABleau II. - Richesse de la rate en trophozoïtes d'attente et en schizontes et richesse de l'intestin en gamétocytes au cours du nycthémère et au cours de l'année.

Les chiffres représentent le nombre de trophozoïtes comptés dans 1000 globules blancs.

\begin{tabular}{|c|c|c|c|c|c|c|c|c|c|c|c|c|c|c|c|}
\hline \multirow{3}{*}{ Heure } & \multicolumn{8}{|c|}{ Trophozoïtes d'attente } & \multicolumn{4}{|c|}{$\begin{array}{l}\text { Schizontes réticulo- } \\
\text { endothéliaux }\end{array}$} & \multicolumn{3}{|c|}{ Gamétocytes } \\
\hline & \multicolumn{2}{|c|}{ Avr. } & \multicolumn{2}{|c|}{ Juil. } & \multicolumn{2}{|c|}{ Nov. } & \multicolumn{2}{|c|}{ Mai } & \multirow[t]{2}{*}{ Avr. } & \multirow[t]{2}{*}{ Juil. } & \multirow[t]{2}{*}{ Nov. } & \multirow[t]{2}{*}{ Mai } & \multirow[t]{2}{*}{ Avr. } & \multirow[t]{2}{*}{ Juil. } & \multirow[t]{2}{*}{ Nov } \\
\hline & $\mathrm{P}$ & G & $\mathrm{P}$ & $\mathrm{G}$ & $\mathbf{P}$ & G & $\mathrm{P}$ & $\mathrm{G}$ & & & & & & & \\
\hline 1 & & & & & & & & & & & & & & & \\
\hline 2 & & & & & & & 2 & 0 & & & & - & & & \\
\hline 3 & 36 & 10 & & & & & & & + & & & & \pm & & \\
\hline 4 & & & & & & & 9 & 0 & & & & - & & & \\
\hline 5 & 44 & 4 & & & 90 & 61 & & & + & & + & 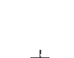 & \pm & & \pm \\
\hline $\begin{array}{l}6 \\
7\end{array}$ & 17 & 0 & & & & & 7 & 0 & + & & & + & \pm & & \\
\hline $\begin{array}{l}8 \\
9\end{array}$ & & & & & & & 17 & 0 & & & & + & & & \\
\hline 10 & & & & & & & & & & & & & & & \\
\hline $\begin{array}{l}11 \\
12\end{array}$ & & & 237 & 8 & 14 & 0 & $\begin{array}{l}20 \\
50\end{array}$ & $\begin{array}{l}0 \\
0\end{array}$ & & ++ & 一 & $\stackrel{+}{-}$ & & ++++ & + \\
\hline 13 & & & 144 & 0 & 24 & 72 & & & & \pm & - & & & ++++ & $+t$ \\
\hline $\begin{array}{l}14 \\
15\end{array}$ & 11 & 3 & & & 8 & 26 & 3 & 0 & + & & + & $\ldots$ & \pm & & + \\
\hline 16 & 5 & 2 & & & 8 & 6 & & & + & & + & & + & & + \\
\hline 17 & & & 404 & 6 & & & & & & \pm & & & & ++++ & \\
\hline 18 & 8 & 1 & & & 64 & 38 & & & - & & - & & + & & \pm \\
\hline 19 & & & 234 & 10 & 7 & 6 & 2 & 1 & & + & + & + & & ++++ & \pm \\
\hline 20 & 13 & 1 & & & & & & & 一 & & & & \pm & & \\
\hline 21 & & & & & 70 & 64 & 2 & 3 & & & + & 一 & & & \pm \\
\hline 22 & 4 & 0 & 504 & 1 & & & & & + & +++ & & & + & +++ & \\
\hline $\begin{array}{l}23 \\
24\end{array}$ & & & & & 48 & 64 & 3 & 0 & & & - & - & & & \pm \\
\hline
\end{tabular}

$\mathrm{P}=$ petits trophozoïtes ; $\mathrm{G}=$ grands trophozoïtes.

- au cours du nycthémère: dans l'ensemble, les stades des différentes espèces sont relativement synchrones : début de la gamétogonie la nuit, augmentation de taille et maturation pendant la journée, formation et excrétion des oocystes l'après-midi.

Les horaires des différentes espèces sont légèrement décalés dans le temps ; ces horaires déterminés sur 2 lots de Moineaux, autopsiés en avril et novembre 1980, sont les suivants (tableau $I I I$ ) :

- en novembre : premiers gamétocytes jeunes mesurant moins de $5 \mu \mathrm{m}$ à $18 \mathrm{~h}$, derniers à $5 \mathrm{~h}$; premiers oocystes intracellulaires à $13 \mathrm{~h}$, derniers à $19 \mathrm{~h} 30$;

- en avril : premiers jeunes gamétocytes de moins de $5 \mu \mathrm{m}$ à $22 \mathrm{~h}$ et derniers à $7 \mathrm{~h}$; premiers oocystes intracellulaires à $16 \mathrm{~h}$, derniers à $22 \mathrm{~h}$. 
TABLEAU III. - Horaires des stades tissulaires intestinaux.

\begin{tabular}{|c|c|c|c|c|c|c|c|c|c|c|c|c|}
\hline Heures & 12 & 14 & 16 & 18 & 20 & 22 & 24 & 2 & 4 & 6 & 8 & 10 \\
\hline $\begin{array}{l}\text { Schizontes dans les } \\
\text { les villosités }\end{array}$ & $\begin{array}{l}\text { Avril } \\
\text { Novembre }\end{array}$ & & & & & & & & & & & \\
\hline $\begin{array}{l}\text { Schizontes dans les } \\
\text { glandes de } \\
\text { Lieberkühn }\end{array}$ & $\begin{array}{l}\text { Avril } \\
\text { Novembre }\end{array}$ & & & & & & & & & & & \\
\hline $\begin{array}{l}\text { Jeunes gamétocytes } \\
<5 \mu \mathrm{m}\end{array}$ & $\begin{array}{l}\text { Avril } \\
\text { Novembre }\end{array}$ & & & & & & & & 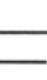 & & & \\
\hline $\begin{array}{l}\text { Oocystes } \\
\text { intracellulaires }\end{array}$ & $\begin{array}{l}\text { Avril } \\
\text { Novembre - }\end{array}$ & & & & & & & & & & & \\
\hline
\end{tabular}

2) Description morphologique

Elle a été faite dans l'article préliminaire (Grulet et coll., 1982).

\section{B - Schizogonies dans l'épithélium des villosités*}

1) Allure générale des infections

- au cours de l'année: nous avons peu de données en été sur la richesse des schizogonies. Un Moineau ( $140 \mathrm{XV}$ ) autopsié à $22 \mathrm{~h} 30$ en juillet était beaucoup plus riche que ceux étudiés en avril ( $431 \mathrm{CL}$ à $20 \mathrm{~h}$ ) et en novembre (705 XV à $19 \mathrm{~h} 30$ ).

- au cours du nycthémère (tableaux III et IV) : les schizontes apparaissent à la tombée de la nuit, peu avant la formation des premiers gamétocytes, et disparaissent lorsque les derniers stades sexués se sont formés entre $5 \mathrm{~h}$ et $7 \mathrm{~h}$.

Les horaires sont déterminés de la même façon que pour la gamétogonie et la sporogonie (cf. plus haut) en avril et en novembre.

- schizogonie C : en avril, début $22 \mathrm{~h}$, fin $3 \mathrm{~h}$; en novembre, début $18 \mathrm{~h}$, fin non observée; sachant que la fin de la schizogonie est peu différente en avril et en novembre, nous pensons qu'elle se situe aux environs de $3 \mathrm{~h}$.

- schizogonie D : en avril, début $22 \mathrm{~h}$, fin $5 \mathrm{~h}$; en novembre, début $18 \mathrm{~h}$, fin $5 \mathrm{~h}$.

- schizogonie $E$ : en avril, début $22 \mathrm{~h}$, fin $3 \mathrm{~h}$; en novembre, début $21 \mathrm{~h} 30$, fin $5 \mathrm{~h}$.

Comme la gamétogonie, les schizogonies débutent donc généralement 4 heures plus tôt en novembre qu'en avril.

2) Description morphologique

Toutes les schizogonies observées dans l'épithélium des villosités sont ectomérogoniques.

- Pour faciliter la compréhension du texte, nous attribuons une lettre (A-B-C-D-E) à chaque type morphologique de schizonte observé dans l'intestin (épithélium des villosités et cryptes de Lieberkühn) des Moineaux; les principaux caractères morphologiques et le siège des schizogonies sont récapitulés dans le tableau $V$. 
TABleau IV. - Différents types de schizontes intestinaux présents au cours du nycthémère en avril et en novembre.

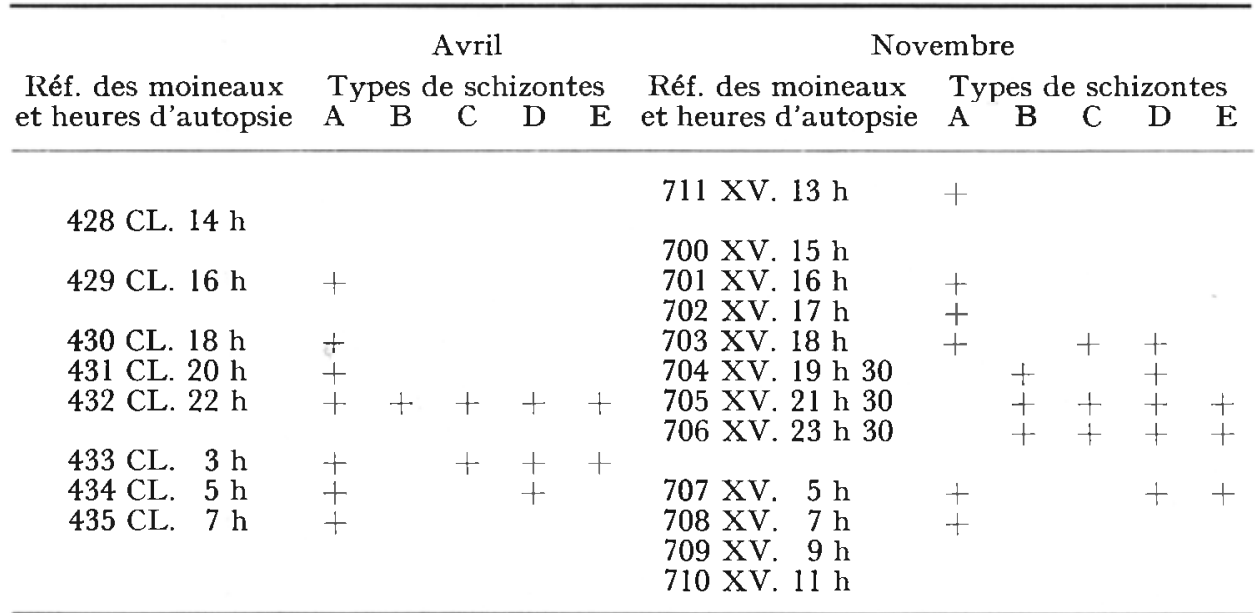

Les croix indiquent la présence de schizontes.

\section{- Schizontes de première génération}

- Observations in vitro :

Des schizontes morphologiquement proches de ceux décrits par Box (1977) comme première génération schizogonique d' $I$. canaria ont été observés dans des cultures primaires de foie de Canari $(P l . I V)$. Ils siègent dans les hépatocytes.

Ils mesurent $16,9 \mu \mathrm{m} \times 12,3 \mu \mathrm{m}$ et produisent à maturité de 15 à 30 mérozoïtes allongés, fins et incurvés; ils mesurent $15,4 \mu \mathrm{m}$ de long et contiennent 2 petits cristalloides de part et d'autre du noyau, qui est médian.

- Observations in vivo:

Le seul parasite observé $(P l . I, 1)$ a encore la forme allongée du sporozoïte ; il est plus grand que le sporozoïte $(15,5 \mu \mathrm{m} \times 4,6 \mu \mathrm{m})$; le noyau est plus grand et plus diffus; le cytoplasme est plus basophile et contient quelques petites vacuoles et 2 cristalloïdes.

- Autres schizogonies

Nous distinguons 3 types morphologiques de schizontes dans l'épithélium des villosités (tableau $V$ ) :

- Type $C(P l . I I, 5,6)$ : Ils siègent tantôt à la base de la cellule épithéliale, où ils font fréquemment saillie entre la basale et l'axe conjonctif, tantôt au sommet et ils ont alors tendance, à maturité, à tomber avec leur cellule-hôte dans la lumière intestinale.

Ils mesurent en coupe de 13 à $15 \mu \mathrm{m}$ de diamètre et produisent un nombre élevé de mérozoïtes (20 à 30), qui paraissent rayonner à partir du centre. Les mérozoïtes 

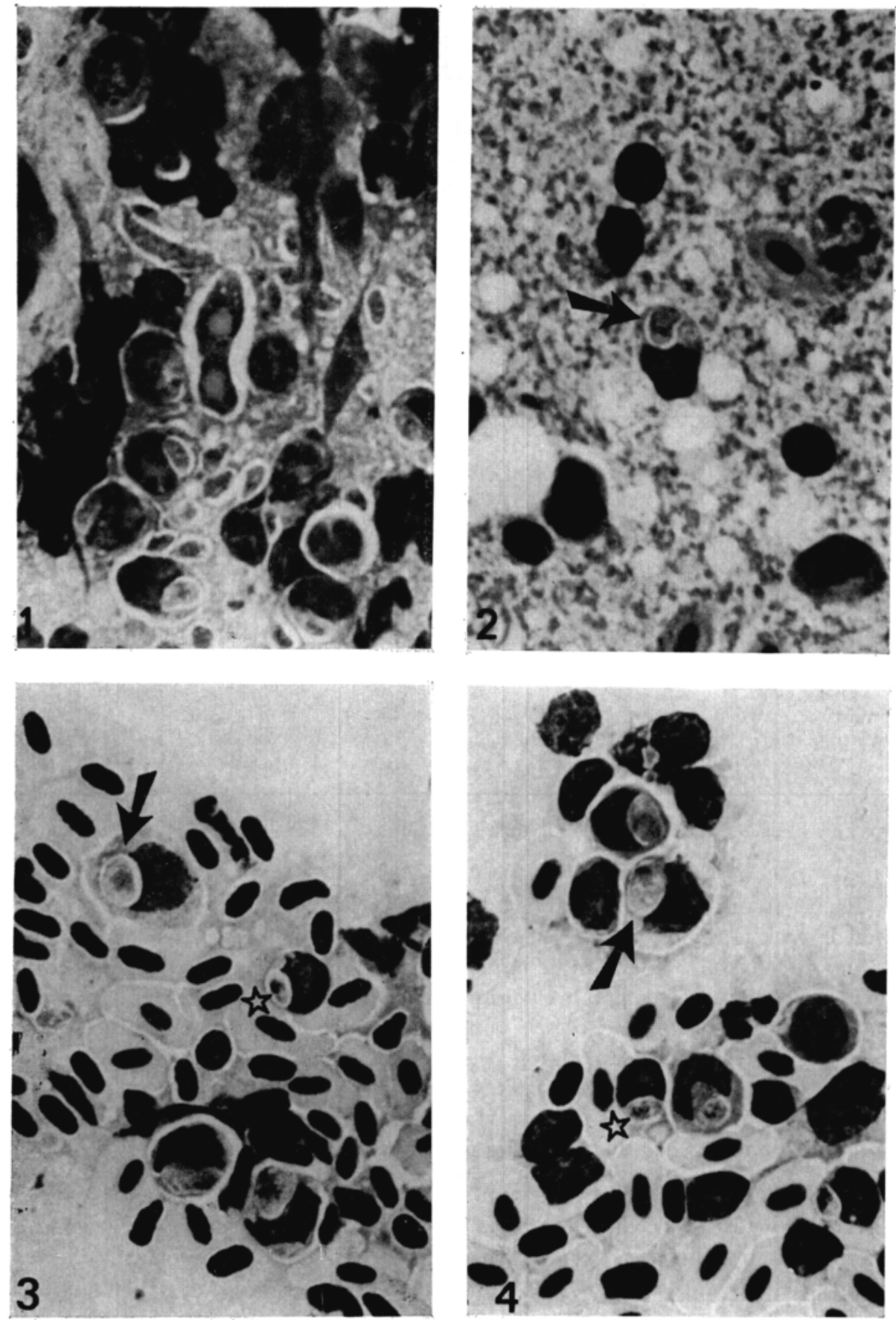

PL. I. - Les deux modes d'évolution du sporozoïte (I et 2) et les trophozoïtes d'attente (3 et 4).

- I : sporozoïte en cours d'évolution vers la schizogonie dans un frottis par apposition d'intestin; il a augmenté de taille sans se diviser en deux, son cytoplasme est devenu très basophile; les deux cristalloïdes sont présents; - 2 : jeune schizonte de première génération d'une espèce à tropisme réticulo-endothélial formé à partir d'un hémi-sporozoïte ; il siège dans un lymphocyte, il est plus petit que le sporozoïte et contient un seul cristallö̈de ; le noyau est fragmenté ; - 3 et 4 : petits trophozoïtes d'attente (étoiles) et grands trophozoïtes (flèches) dans des frottis par apposition de rate. I ( $X$ I 400$) ; 2,3$ et 4 ( $X$ I 0oo). 
mesurent $9 \mu \mathrm{m} \times 3 \mu \mathrm{m}$ en apposition, leur cytoplasme est assez basophile, avec un noyau central peu dense ; leurs deux extrémités sont pointues, et le complexe apical, à une extrémité, est fréquemment coloré en rose.

- Type $D(P l . I I, 3,4)$ : Comme le type $\mathrm{C}$, ils peuvent siéger à la base ou au sommet de la cellule et, dans ce dernier cas, contrairement au schizonte de type $C$, ils ne sont pas entraînés vers la lumière.

Tableau V. - Principaux caractères différentiels des schizontes intestinaux.

\begin{tabular}{cccccc}
\hline $\begin{array}{c}\text { Types de } \\
\text { schizontes }\end{array}$ & A & B & C & D & E \\
\hline $\begin{array}{c}\text { Siège } \\
\begin{array}{c}\text { Nombre de } \\
\text { mérozoïtes }\end{array}\end{array}$ & CL 20 & CL & V & V & V \\
$\begin{array}{c}\text { Forme des } \\
\text { mérozoïtes } \\
\begin{array}{c}\text { Taille des } \\
\text { mérozoïtes } \\
\text { en apposition } \\
\text { (en } \mu \mathrm{m})\end{array}\end{array}$ & allongés & allongés & allongés & allongés & $\begin{array}{c}\text { arrondis ou } \\
\text { piriformes }\end{array}$ \\
\hline
\end{tabular}

CL : épithélium des cryptes de Lieberkühn. - V : épithélium des villosités.

Ils mesurent en coupe $14 \mu \mathrm{m} \times 10 \mu \mathrm{m}$ et, à maturité, contiennent 3 à 12 mérozoites de grande taille, disposés en quartiers d'orange et mesurant en apposition 9 à $15 \mu \mathrm{m}$ de long sur 3 à $4,5 \mu \mathrm{m}$ de large. Les mérozoïtes ont un cytoplasme très fortement basophile, avec de nombreux grains azurophiles ; le noyau est grand et dense. En apposition, ils ont une extrémité pointue et l'autre plus arrondie, coiffée par une masse rose correspondant au complexe apical. Certaines variations morphologiques dans la taille et le nombre de mérozoïtes existent en fonction de l'heure et du niveau de l'intestin. Ces variations sont liées vraisemblablement à des morphologies un peu différentes d'une espèce à l'autre. Elles ne peuvent donc pas être analysées.

- Type E (Pl. II, 1,2) : Comme pour les types précédents, ils siègent tantôt audessus du noyau de la cellule, tantôt au-dessous ; dans ce dernier cas, ils soulèvent la basale et font saillie dans l'espace entre l'épithélium et l'axe conjonctif.

Ils mesurent en coupe de 13 à $14 \mu \mathrm{m}$ de diamètre. Les mérozoïtes, peu nombreux (8 à 12), se forment, par bourgeonnement, à la périphérie d'une masse cytoplasmique, qui disparaît à maturité. Lorsque le schizonte est mûr, ils sont libres à l'intérieur d'une grande vacuole claire. Les mérozoïtes, arrondis ou piriformes, mesurent en apposition $3 \mu \mathrm{m}$ à $4,5 \mu \mathrm{m}$. Leur cytoplasme est clair et leur noyau, de grande taille, occupe $1 / 3$ du volume du mérozoite. 

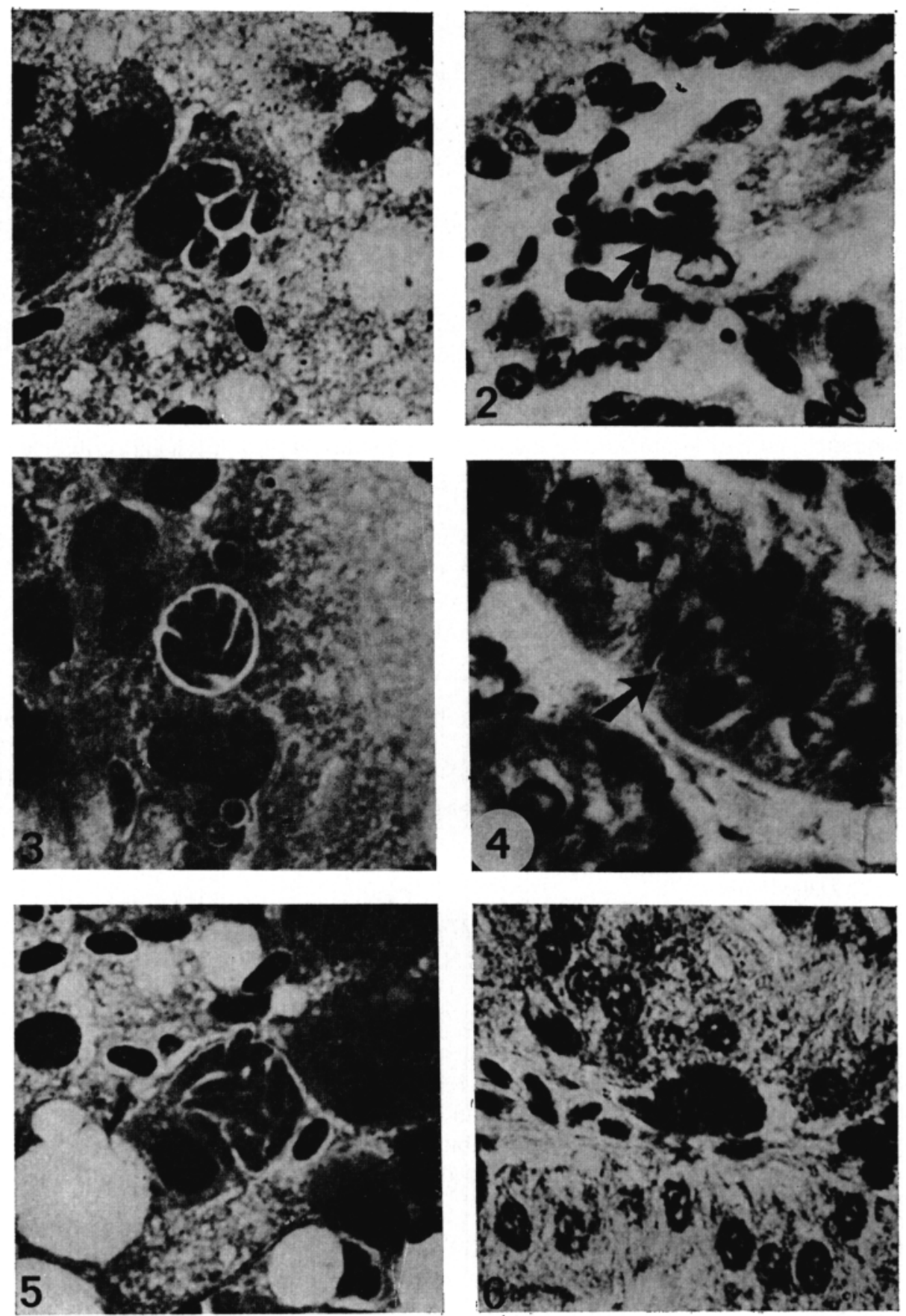

PL. II. - Schizontes dans I'épithélium des villosités ;

I, 3 et 5 : frottis par apposition; 2,4 et 6 : coupes.

-- I et 2 : schizontes de type $\mathrm{E} ;-3$ : schizontes de type $\mathrm{D} ;-4$ : schizonte de type $\mathrm{D}$ siégeant au-dessus du noyau de la cellule-hôte ; $-5:$ schizonte de type $\mathrm{C} ;-6:$ schizonte de type C siégeant sous le noyau de la cellule-hôte et faisant saillie vers l'axe conjonctif ( $X$ I $O \circ)$. 


\section{C - Schizogonies dans les glandes de Lieberkühn}

1) Allure générale de l'infection

- au cours de l'année : deux catégories de schizontes sont observées dans les glandes de Lieberkühn : les uns ne sont présents qu'en été et, dans notre interprétation (cf. plus loin), représentent des stades faisant directement suite à l'ingestion des oocystes ; les autres (schizontes A et B) sont observés toute l'année.

- au cours du nycthémère :

En été, pendant la période de transmission, les deux types de schizogonies se chevauchent et il n'est pas possible d'étudier leur périodicité.

En hiver, seuls les schizontes A et B sont présents.

Ils sont observés à presque toutes les heures de la journée (tableau $I V$ ) ; ils sont abondants pendant la nuit (de $18 \mathrm{~h}$ à $7 \mathrm{~h}$ en novembre et $20 \mathrm{~h}$ à $7 \mathrm{~h}$ en avril) et très rares pendant la journée.

Nous ne trouvons pas de schizontes dans les glandes de Lieberkühn lors des autopsies effectuées à $9 \mathrm{~h}$ et $11 \mathrm{~h}$ en novembre, et à $14 \mathrm{~h}$ en avril au moment où l'infection lieberkühnienne est faible. Par contre, quelques schizontes A et B sont observés chez d'autres Oiseaux sacrifiés aux mêmes heures en juillet.

Ils ont les horaires suivants (tableau $I V$ ) :

- type $A$ : présent tout au long des 24 heures;

- type $B$ : en avril, début $22 \mathrm{~h}$, fin non observée (? vers $2 \mathrm{~h}$ ) ; en novembre, début 19 h 30, fin non observée (? vers 4 h).

La fin de la schizogonie B n'a pu être déterminée avec présision, mais, dans notre interprétation (cf. plus loin), elle est à l'origine de la schizogonie $\mathrm{E}$ et doit donc se terminer peu avant celle-ci.

\section{2) Description}

- Schizontes présents uniquement en été $(P l . I I I, 1,2)$ : ils sont décrits chez 3 Moineaux : $683 \mathrm{CL}$ autopsié à $9 \mathrm{~h}$ en juin, $358 \mathrm{XI}$ à $10 \mathrm{~h}$ en juillet et $131 \mathrm{XV}$ à $11 \mathrm{~h} 30 \mathrm{en}$ août.

Ils ont un nombre élevé de mérozoïtes (entre 20 et 30) et siègent dans une cellulehôte qui a généralement été entraînée vers la lumière de la glande. Les uns ont des mérozoïtes piriformes, mesurant en coupe $3 \mu \mathrm{m} \times 2 \mu \mathrm{m}$, rayonnant à partir du centre. Les autres ont des mérozoites de plus grande taille $(4,5 \mu \mathrm{m} \times 3 \mu \mathrm{m})$, arrondis, avec un grand noyau dont le nucléole bien visible est entouré par une zone claire et arrondie. Le noyau de la cellule hôte dans les deux cas est plus condensé que celui des cellules voisines.

- Schizontes présents toute l'année (tableau V):

Les types $\mathrm{A}$ et $\mathrm{B}$ ont certains caractères communs, qui sont :

- leur siège, tantôt à la base de la cellule hôte, tantôt au sommet; dans ce dernier cas, ils ont tendance à tomber dans la lumière ;

- leur nombre de mérozoïtes, qui est élevé : supérieur à 20 ; 
- les modifications des noyaux de leurs cellules hôtes, qui sont tantôt hypertrophiés et clairs, tantôt au contraire contractés et denses.

Les caractères particuliers des schizontes de type A et B sont les suivants :

- Type $A(P l . I I I, 3)$ : 20 à 50 mérozoïtes de petite taille $(6 \mu \mathrm{m} \times 1,5$ à $2 \mu \mathrm{m}$ en apposition), allongés et fins, au cytoplasme peu chromophile ; ils possèdent un petit complexe apical à une extrémité.

- Type $B(P l . I I I, 4): 20$ à 30 mérozoïtes plus grands $(9 \mu \mathrm{m} \times 3 \mu \mathrm{m}$ en apposition) et plus trapus que les précédents, au cytoplasme fortement basophile ; ils possèdent un complexe apical relativement grand ; ils sont morphologiquement très proches des mérozoïtes du type $\mathrm{C}$ de l'épithélium des villosités.

\section{D - Stades dans le système réticulo-endothélial}

\section{1) Allure générale de l'infection}

Des stades d'Isospora siégeant dans le système réticulo-endothélial sont trouvés chez tous les Moineaux examinés, soit au moment de leur capture, soit après plusieurs semaines de séjour dans l'animalerie.

Ce sont : des schizontes ectomérogoniques; des schizontes endomérogoniques ; des mérozoïtes libérés par les schizontes; des trophozoïtes.

Chez les Moineaux, on rencontre de nombreux trophozoïtes dont les caractères morphologiques sont ceux d'un parasite au développement arrêté : noyau au repos, cytoplasme très peu dense ; nous les désignons sous le nom de "trophozoïtes d'attente ".

Deux populations de trophozoïtes de ce type sont observées; l'une, de petite taille $(4 \mu \mathrm{m})$, l'autre, de grande taille $(7,5 \mu \mathrm{m})$. Lorsque ces deux populations sont présentes et abondantes en même temps, on observe des stades intermédiaires entre les deux. Dans la suite de l'exposé, nous utiliserons les termes de petits et grands trophozoïtes d'attente.

A un moment déterminé, les Oiseaux capturés dans un même biotope ont une infection d'intensité comparable et les stades observés sont semblables d'un Oiseau à l'autre.

Schématiquement, on rencontre deux types d'infection :

- des infections chroniques, qui représentent la grande majorité des cas : seuls des trophozoïtes en attente (petits et grands) très nombreux et rarement quelques schizontes endomérogoniques sont observés ;

- des infections aiguës : de nombreuses formes de multiplication prolifèrent dans les organes; nous observons des schizontes ectomérogoniques et endomérogoniques, de nombreux mérozoïtes intra- et extracellulaires et une majorité de petits trophozoïtes d'attente.

\section{a) Variation au cours de l'année}

Les infections chroniques avec une majorité de trophozoïtes d'attente s'observent pendant les mois froids d'octobre à mars. 

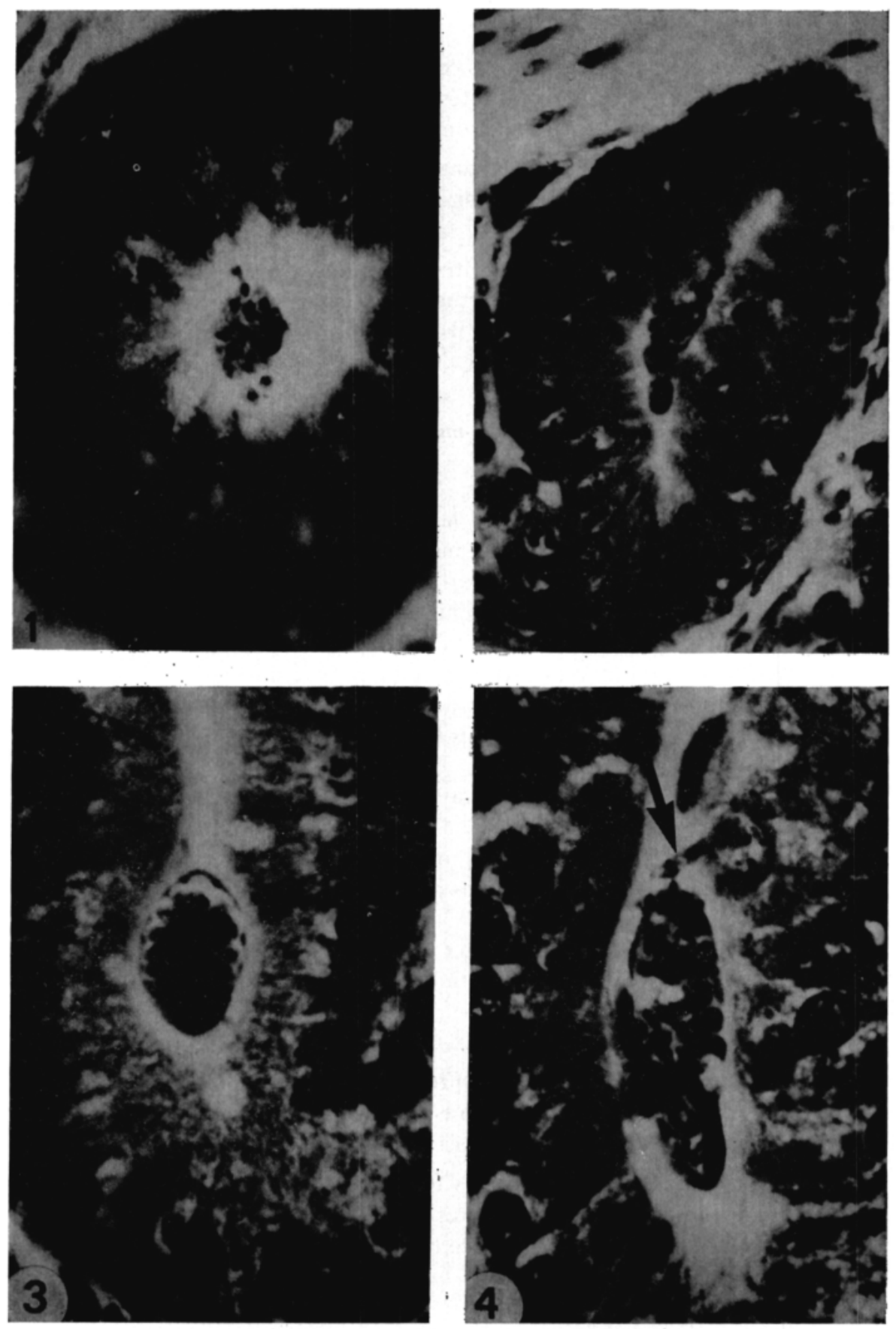

PL. III. - Schizontes en coupe dans les glandes de Lieberkühn.

Ils sont tous mûrs et sont tombés dans la lumière de la glande.

- I et 2 : schizontes observés uniquement pendant la période de transmission; sur la photo I le noyau de la cellule-hôte, plus condensé que la normale est visible $;-3:$ schizonte de type $A ;-4:$ schizonte de type $B$ : noter le mérozoilte (flèche) en coupe longitudinale ( $X$ I $\circ \circ)$ ). 
Les infections aiguës sont toujours décelées en été de juin à septembre; le nombre le plus élevé de schizontes est trouvé chez des Moineaux capturés en juillet (tableau $I I$ ).

L'endomérogonie est la forme de division la plus fréquemment observée ; ncus ne trouvons de l'ectomérogonie que chez 5 Moineaux capturés en juillet.

\section{b) Étude de l'infection réticulo-endothéliale au cours des 24 heures}

Elle est étudiée dans 4 séries de Moineaux autopsiés à intervalles de temps rapprochés pendant 24 heures : en avril, juillet et novembre 1980 et en mai 1981 (cf. matériel et méthodes).

Les résultats figurent dans le tableau $I I$.

Aucune recrudescence de la schizogonie n'apparaît au cours des 24 heures et les différences observées dans le nombre de trophozoïtes nous paraissent être liées à des variations individuelles plutôt qu'à l'horaire de l'autopsie.

c) Étude de l'infection sanguine pendant 1 semaine

L'étude journalière des trophozoïtes sanguins d'un Moineau (708 MB), capturé en avril 1982, est faite matin et soir pendant une semaine. Sur des frottis sanguins effectués après ponction de la veine de l'aile, nous comptons les trophozoïtes dans les mononucléaires (tableau VI).

Il ne semble pas y avoir de variation significative de la richesse en trophozoïtes sanguins du matin au soir et d'un jour à l'autre.

\section{Tableau VI. - Nombre de trophozoites dans les frottis de sang. \\ (Les chiffres représentent le nombre de trophozoïtes dans 1000 globules blancs examinés).}

\begin{tabular}{ccc}
\hline Jours & Matin & Soir \\
\hline 1 & 35 & 31 \\
2 & 18 & 25 \\
3 & 34 & 38 \\
4 & $\overline{31}$ & 11 \\
5 & 12 & 18 \\
6 & 12 & 15 \\
7 & & \\
\hline
\end{tabular}

\section{2) Description}

a) Schizogonies réticulo-endothéliales

- Schizontes ectomérogoniques

- Schizontes de $1^{\text {re }}$ génération

Ils sont reconnaissables à la présence de cristalloïdes.

- in vitro $(P l . I V)$ : leur développement complet a été observé; ils siègent tantôt 

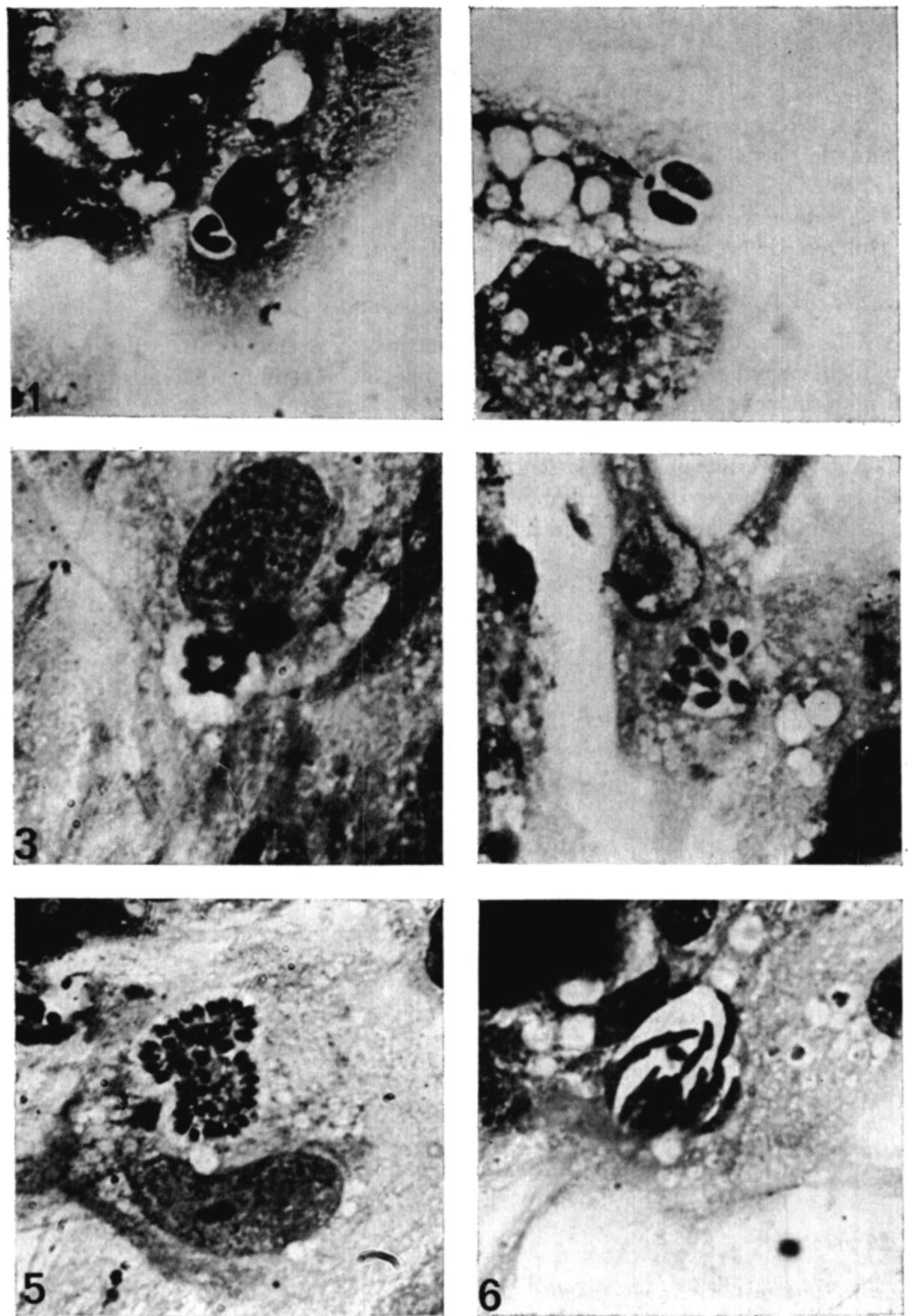

PL. IV. - Stades obtenus en cultures primaires de cellules de foie de Canari.

I à 5 : cycle à tropisme réticulo-endothélial ; $6:$ cycle de type $I$. canaria.

- I : début de la division du sporozoïte en deux; une constriction apparaît à la partie médiane du sporozoïte (deux heures de culture) ; - 2 : sporozoïte divisé en deux parties égales et un petit corps résiduel (flèche) (2 heures de culture); - 3: schizonte de première génération ; noter le petit cristalloïde dans chaque mérozoïte (24 heures de culture) ; - 4 : schizonte de première génération; noter le petit corps résiduel ( 24 heures de culture) ; - 5: schizontes ectomérogoniques a petits mérozoïtes ( 7 jours de culture) ; - 6 : schizonte de première génération du cycle de type I. canaria (I 8 heures de culture). I, 3, 5 et 6 (X I 400); 2 et 4 ( $\times$ I 00o). 
dans un hépatocyte, tantôt dans un fibroblaste; ils se forment à partir d'un hémisporozoïte (Millet et coll., 1984) ; le noyau se divise et les noyaux fils forment une couronne autour d'un cristalloïde ; les mérozoïtes bourgeonnent à la périphérie ; ils sont au nombre de 8 à 12 ; à maturité, le cristalloïde initial s'est fragmenté et chaque mérozoïte contient un petit cristalloïde proche du noyau.

- in vivo $(P l . I, 2)$ : nous avons observé deux schizontes de ce type tous deux immatures avec un grand cristalloïde central entouré par des noyaux; ils mesurent $4,5 \mu \mathrm{m} \times 3 \mu \mathrm{m}$ et siègent dans la rate d'un Moineau capturé en avril 1980 .

Box, en 1977, n'a pas observé la division en deux du sporozoïte d'I. serini, mais elle décrit, dans les tissus du Canari infecté expérimentalement des "sporozoites» beaucoup plus petits que ceux de l'oocyste et ne contenant qu'un seul cristalloïde.

Il est donc vraisemblable que le phénomène de division en deux du sporozoïte se produise également chez Isospora serini.

- Autres schizontes ectomérogoniques ( $P l . V, 1$ et 2)

Nous les décrivons chez des Moineaux capturés en juillet 1980. Ils siègent préférentiellement dans le foie et le conjonctif de l'intestin. Les formes immatures sont des masses arrondies au cytoplasme très basophile, vacuolé ; les noyaux, grands, irréguliers, à chromatine grossière, sont d'abord uniformément distribués, puis ils deviennent périphériques et forment une couronne régulière. Les mérozoïtes bourgeonnent à partir d'une masse cytoplasmique centrale dense et basophile. Ils sont au nombre de 8 à 12 et entourent le corps résiduel cytoplasmique. L'ensemble mesure alors $8,5 \mu \mathrm{m} \times 7 \mu \mathrm{m}$, en apposition. Les mérozoïtes libérés sont petits, minces et incurvés; ils mesurent en apposition 3,1 à $4,6 \mu \mathrm{m} \times 1,5$ à 2,2 $\mu \mathrm{m}$. Il n'y a pas de cristalloïde.

- Schizontes endomérogoniques ( $P l . V, 3$ à 6 )

Ils siègent préférentiellement dans la rate et le conjonctif intestinal, dans des cellules jeunes à grand noyau, au cytoplasme très basophile, que nous n'avons pas pu identifier. Parfois, les schizontes se trouvent dans des grands lymphocytes.

Ils sont très polymorphes.

Les schizontes de grande taille, encore immatures, présentent des incisures dans le cytoplasme, délimitant des îlots nucléocytoplasmiques, tantôt inégaux, mais le plus souvent égaux entre eux et contenant 2 noyaux; les mérozoïtes se forment à l'intérieur de la masse cytoplasmique, où l'on note quelques résidus irréguliers de cytoplasme, qui disparaissent lorsque le schizonte est complètement mûr.

Le schizonte mûr a une taille de $11,5 \mu \mathrm{m} \times 6 \mu \mathrm{m}$ et contient un nombre très variable de mérozoïtes (de 2 à 30 ) ; dans de nombreux cas, ceux-ci ne sont pas libérés dès leur formation, et l'on observe des accumulations de mérozoïtes dans une même enveloppe.

Il n'est pas rare d'observer des petits schizontes à 2-3 et 4 mérozoïtes groupés dans une même cellule ; lorsque les mérozoïtes ne sont pas complètement séparés, on distingue très nettement des scissures longitudinales entre eux $(P l . V, 3)$.

Les mérozoïtes sont renflés à leur partie médiane et pointus aux deux extrémités ; ils mesurent en apposition $4,6 \mu \mathrm{m} \times 1,5$ à $2,2 \mu \mathrm{m}$. 

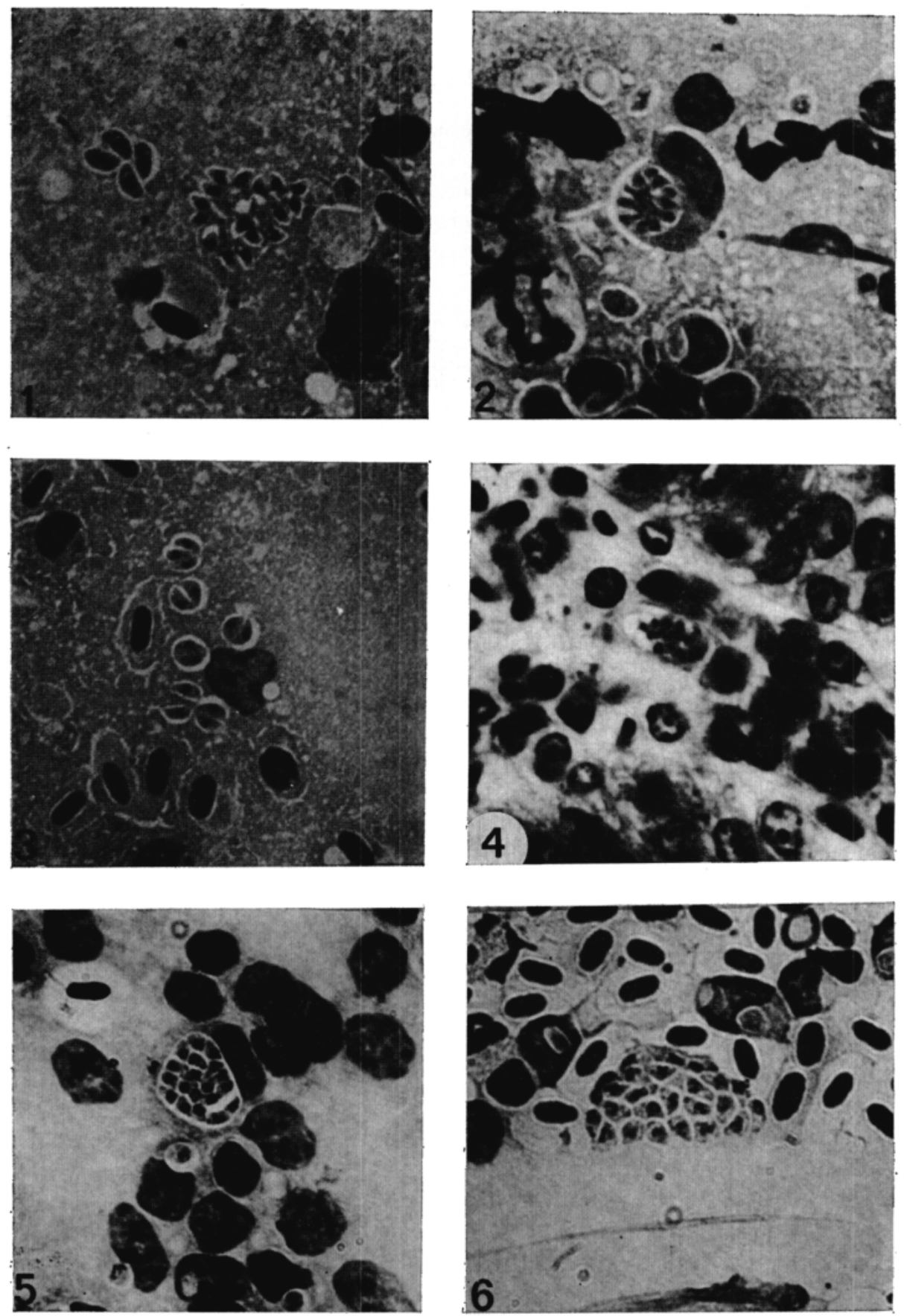

Pr. V. - Schizogonies dans le système réticulo-endothélial :

I, 2, 3, 5 et 6 : frottis par apposition; 4 : coupe.

- I : schizonte ectomérogonique dans le foie; - 2 : schizonte ectomérogonique dans l'axe conjonctif des villosités intestinales ; - 3: groupement de petits schizontes endomérogoniques à 2 mérozoïtes ; la cellule-hôte, qui est un mononucléaire du foie est endommagée lors de la confection du frottis; - 4 : schizonte mûr endomérogonique dans l'axe conjonctif d'une villosité ; - 5: schizonte endomérogonique presque mûr dans la rate ; certains noyaux sont encore reliés entre eux par le cytoplasme; - 6: schizonte endomérogonique mûr dans la rate. I, 2, 5 et 6 $\times$ I 400$) ; 3$ et 4 (X I 000). 
b) Trophozoïtes en attente dans le système réticulo-endothélial ( $P l . I, 3$ et 4)

Il n'est pas possible, en l'absence d'une étude ultrastructurale, de déterminer de façon indiscutable le moment où le mérozoïte devient trophozoïte. Cependant, nous jugeons que, lorsque ses extrémités se sont arrondies, il a commencé son évolution et doit être considéré comme trophozoïte.

Les petits trophozoïtes siègent de préférence dans les petits lymphocytes et les grands trophozoïtes dans les lymphocytes de plus grande taille; les autres cellules mononucléées sont rarement envahies; les polynucléaires sont indemnes.

Tous les tissus sont atteints, mais à des degrés divers.

La rate est le tissu le plus riche en petits et grands trophozoïtes, suivie par le foie ; le rein et la moelle osseuse, en particulier, sont relativement pauvres et hébergent surtout des petits trophozoites. Contrairement aux observations de Box, qui note une accumulation de trophozoïtes d'I. serini dans le poumon, nous trouvons toujours cet organe pauvre en parasites.

- Les petits trophozoïtes sont issus des mérozoïtes endomérogoniques; ils sont ovalaires $(3,2 \times 4,5 \mu \mathrm{m})$, ou ronds (diamètre $4,8 \mu \mathrm{m}$ ), avec un cytoplasme très pâle et un noyau de granules chromatiniens assez grossiers, entourés par un espace clair.

- Les grands trophozoïtes mesurent environ 7,5 $\mu \mathrm{m}$; ils sont arrondis, ovalaires et plus rarement allongés ; le cytoplasme est bleu clair ou gris et contient fréquemment des granules azurophiles; le noyau est rose pâle, mal limité, avec quelques granules chromatiniens fins.

Tous les intermédiaires peuvent être observés entre les petits et les grands trophozoïtes.

\section{E - Interprétation des cycles}

Pour rendre compte de l'ensemble des faits observés, il apparaît que ces espèces, très proches par la morphologie des oocystes, évoluent néanmoins selon des cycles différents.

Trois schémas de cycles sont proposés :

1 - cycle de durée limitée à quelques jours, évoluant seulement dans l'épithélium des villosités ;

2 - cycle prolongé durant au moins une année. Ce cycle présente d'une part, les stades habituels de l'épithélium des villosités (schizogonie, gamétogonie, sporogonie), d'autre part une schizogonie dans les glandes de Lieberkühn. Cette schizogonie est peu abondante au cours de la journée et devient intense pendant la nuit ;

3 - cycle prolongé, durant au moins une année. Ce cycle présente, d'une part, des stades évoluant, selon le schéma classique, dans l'épithélium des villosités, d'autre part, toute une série de formes envahissant le système réticulo-endothélial des autres tissus (schizontes et trophozoïtes d'attente). 
1) Cycle court de type Isospora canaria

Le schéma proposé est élaboré à partir de la description d'I. canaria par Box (1977).

a) Schéma du cycle (fig. 1)

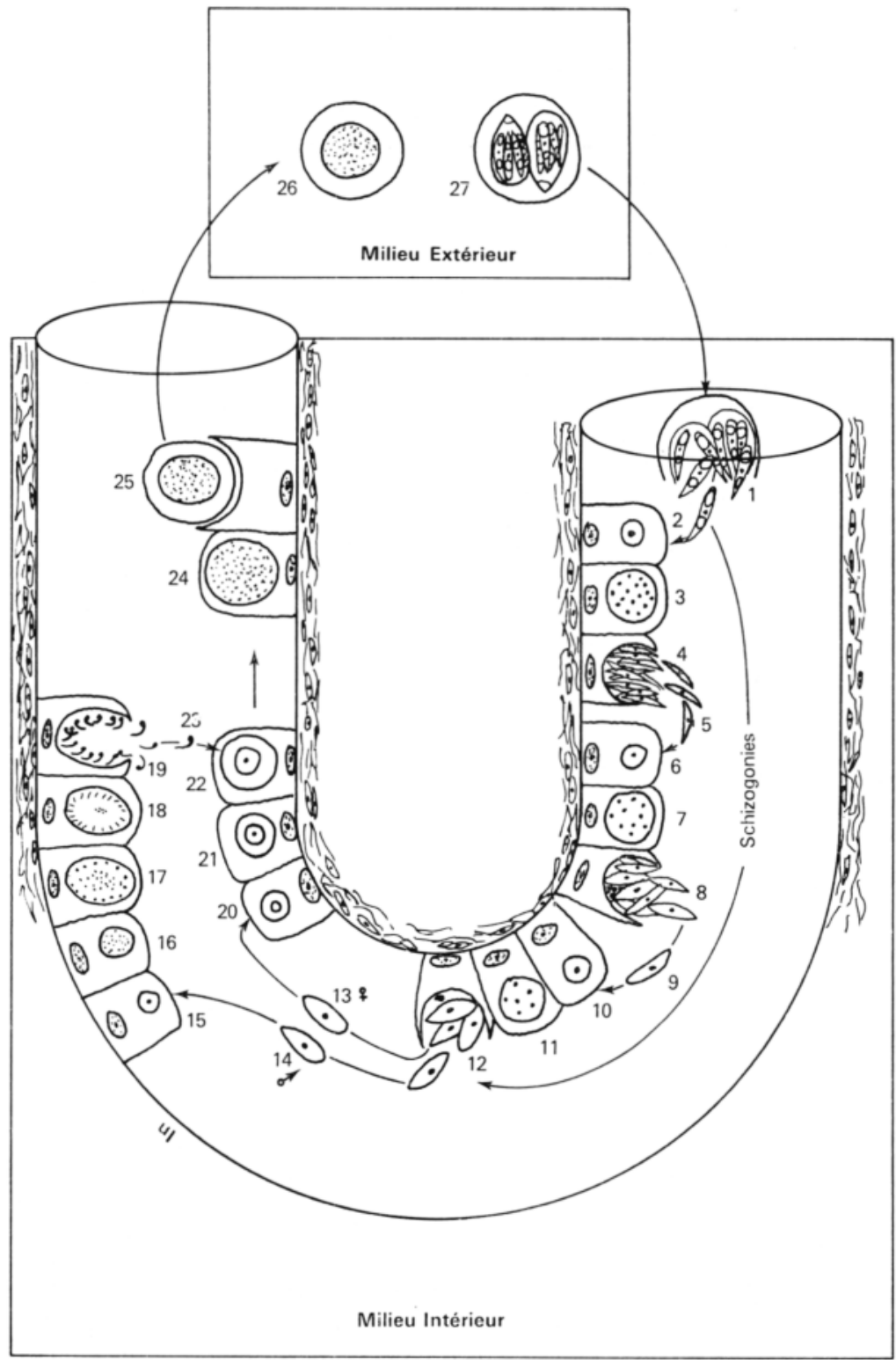

FIg. I. - Schéma du cycle d'I. canaria, adapté de Box (I977). 
L'oocyste (1) s'ouvre dans l'intestin de l'hôte et libère des sporozoïtes qui pénètrent dans une cellule des villosités (2) où ils se divisent (3) pour donner un schizonte (4). Les mérozoïtes issus de ce schizonte (5) pénètrent dans une autre cellule (6) pour donner naissance à une nouvelle schizogonie $(7,8)$.

Les mérozoïtes de ce schizonte (9) pénètrent dans une autre cellule des villosités (10) et se divisent (11) pour donner un $3^{\mathrm{e}}$ schizonte (12). Les mérozoïtes issus de cette dernière schizogonie (13 et 14) sont à l'origine de la gamétogonie mâle (15 à 19) et femelle (20 à 22). A maturité, les gamètes mâles (23) quittent la cellule où ils se sont formés et fécondent les gamètes femelles (22). L'oocyste ainsi formé (24) quitte sa cellule hôte et tombe dans la lumière intestinale (25) ; il sera excrété dans le milieu extérieur, où il mûrit $(26,27)$ en quelques jours, avec formation de deux sporocystes contenant chacun quatre sporozoïtes.

\section{- Cycle nycthéméral}

Seul l'horaire d'excrétion des oocystes est étudié par Box : cet auteur observe des oocystes entre $14 \mathrm{~h}$ et $8 \mathrm{~h}$ le lendemain, mais aucune étude précise n'est faite au cours de la nuit et il est vraisemblable que les oocystes observés le matin sont émis la veille au soir.

- Durée d'évolution des phases du cycle

Période prépatente : 4 à 5 jours ;

Période patente : 12 jours.

\section{b) Discussion}

Les stades de ce cycle ne sont pas identifiés in vivo, mais nous obtenons en culture primaire de foie de Canari (cf. plus haut), à partir de sporozoïtes d'Isospora de Moineaux, des schizontes morphologiquement identiques à ceux de la première génération schizogonique d'I. canaria; la morphologie de ces stades présente suffisamment de particularités pour que l'on puisse les considérer comme équivalents chez le Moineau et le Canari.

\section{2) Cycle prolongé à tropisme lieberkühnien}

\section{a) Schéma du cycle (fig. 2)}

L'oocyste (32), lorsqu'il est ingéré par le Moineau, s'ouvre dans le tube digestif (1) ; le sporozoïte libéré (2) est pourvu de 2 cristalloïdes et pénètre dans une cellule des glandes de Lieberkühn, où il grandit (3), puis il subit une $1^{\text {re }}$ division schizogonique (4) et (5) ; les mérozoïtes (6) pénètrent dans une nouvelle cellule des glandes de Lieberkühn et donnent naissance au schizonte de $2^{\mathrm{e}}$ génération de la période prépatente (7) ; après leur libération, les mérozoïtes (8) envahissent de nouvelles cellules lieberkühniennes où ils subissent $n$ schizogonies successives (9 à 13) de type $A$; au début de la nuit, ces schizontes (13) donnent naissance tantôt à une nouvelle schizogonie de type $\mathrm{A}$ (9), tantôt à une schizogonie de type $\mathrm{B}$ (15). La schizogonie (15) libère des mérozoïtes (16) qui envahissent une cellule de l'épithélium des villosités pour donner un schizonte de type E (17). Les mérozoïtes (18) et (22) sont à l'origine des gamétocytes (19) et (23). Le reste du cycle $(20,21$ et 25 à 32) est conforme à ce qui a été décrit plus haut (cf. cycle de type $I$. canaria). 


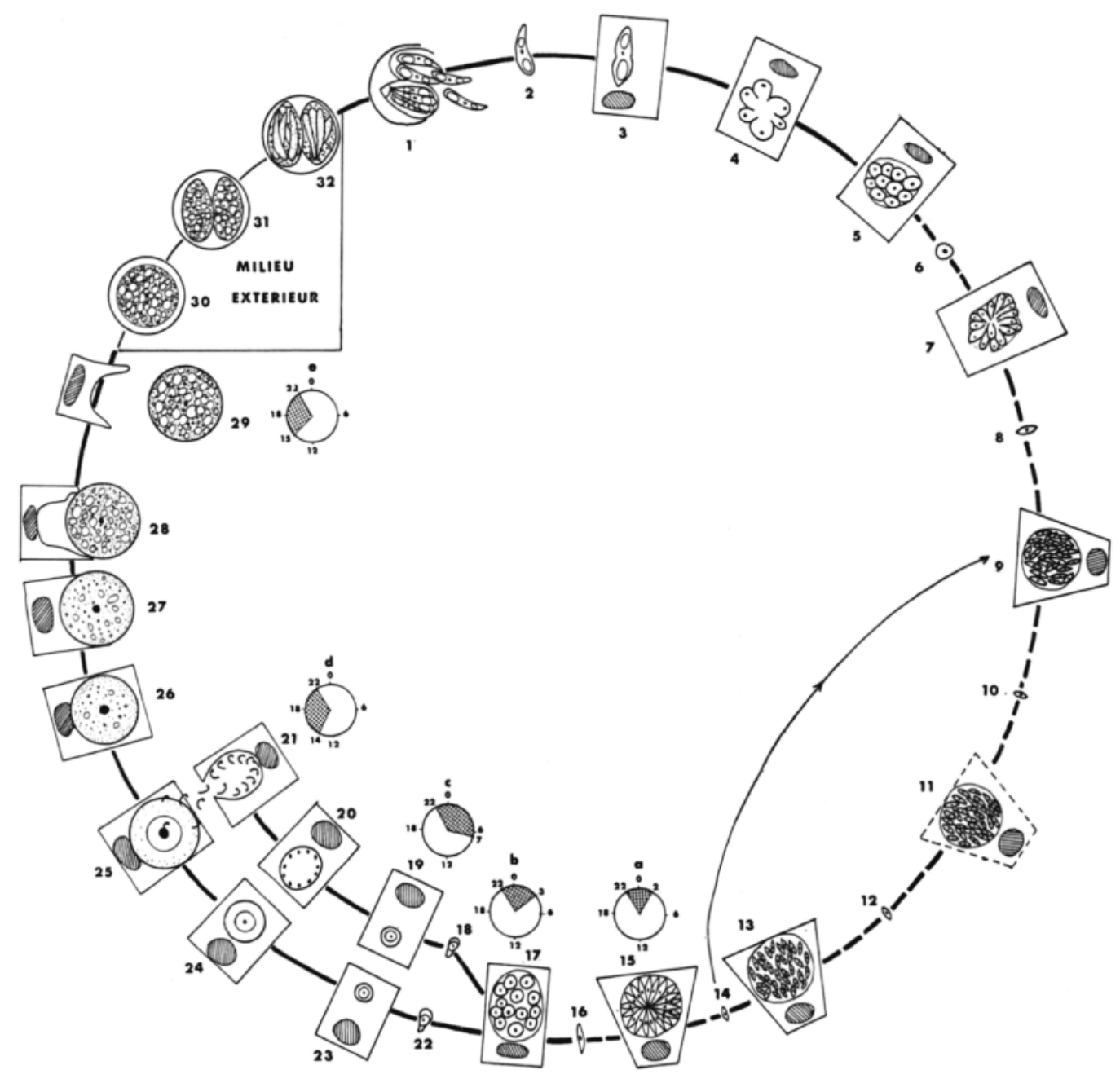

FIG. 2. - Cycle à tropisme Lieberkühnien.

\section{- Cycle nycthéméral (tableaux III et IV)}

Il varie de quelques heures selon la période de l'année; en avril, les horaires sont les suivants :

- schizogonie 15 (type B) : de $22 \mathrm{~h}$ à $3 \mathrm{~h}$;

- schizogonie 17 (type E) : de $22 \mathrm{~h}$ à $3 \mathrm{~h}$;

- jeunes gamétocytes (19 et 23 ) : de $22 \mathrm{~h}$ à $7 \mathrm{~h}$;

- gamétocytes mûrs (21 et 25); oocystes intracellulaires (26-28) et oocystes dans la lumière de l'intestin (29): ces stades sont concomitants de $14 \mathrm{~h}$ à $22 \mathrm{~h}$. 
- Durée d'évolution des phases du cycle

- période prétatente : inconnue ;

- cycle journalier dans l'épithélium des villosités (17 à 29) : $24 \mathrm{~h}$;

- cycle dans les glandes de Lieberkühn : chronique; schizogonie abondante la nuit et rare le jour ;

- période patente : infection chronique;

- maturation des oocystes dans le milieu extérieur : elle varie avec la température : $72 \mathrm{~h}$ à $23^{\circ} \mathrm{C}$.

b) Discussion

Nous avons des observations directes des stades 4 à 32 .

- Modalités de division du sporozoite. Nous ignorons si elle s'effectue : selon le type classique d'I. canaria, où le sporozoïte donne directement naissance à un schizonte, ou selon le type des Isospora à tropisme réticulo-endothélial (cf. plus loin), où le sporozoïte subit une première division en deux moitiés. A titre provisoire, nous considérons que le sporozoïte se transforme directement en schizonte, ce qui est conforme à ce que l'on observe dans la majorité des autres cycles de Coccidies connus.

- Rattachement de la schizogonie 17 dans les villosités intestinales au cycle à tropisme lieberkühnien

Quatre types de schizontes apparaissent dans l'intestin à la tombée de la nuit avant le début de la gamétogonie (types B, C, D, E), et cela même lorsque les formes à cycle court sont absentes ou présentes en quantité négligeable.

Les schizontes de type $B$ lieberkühnien et $C$ dans les villosités sont morphologiquement proches (cf. description) : ils sont à l'origine des schizontes $\mathrm{E}$ et $\mathrm{D}$, qui précèdent l'apparition des gamétocytes.

En rassemblant les documents disponibles d'avril et de novembre, les heures d'apparition et de disparition des quatre formes sont les suivantes :

$\begin{array}{llc} & \text { Apparition } & \text { Disparition } \\ \text { B } & 19 \overline{\mathrm{h}} 30 & \text { avant } 3 \mathrm{~h} \\ \mathrm{C} & 18 \mathrm{~h} & 3 \mathrm{~h} \\ \mathrm{D} & 18 \mathrm{~h} & 5 \mathrm{~h} \\ \mathrm{E} & 21 \mathrm{~h} 30 & 3 \mathrm{~h}\end{array}$

L'étude morphologique montre des formes intermédiaires entre les prégamétocytes et les schizontes D, d'une part, E, d'autre part. E et D sont donc postérieurs à $B$ et $C$, ce qui par ailleurs est satisfaisant, puisque $B$ et $C$ se ressemblent, alors que $\mathrm{D}$ et $\mathbf{E}$ sont clairement différents. Étant donné les heures d'apparition et de disparition, nous devons donc séparer une lignée lieberkühnienne $\mathrm{B}$ et $\mathrm{E}$, et une lignée réticulo-endothéliale $\mathrm{C}$ et $\mathrm{D}$.

Nous rattachons donc les schizontes de type B (15) et E (17), apparus les derniers, au cycle à tropisme lieberkühnien.

- Succession des stades

Ne sont traités ici que les stades prêtant à discussion. Les phases classiques telles que la gamétogonie et la sporogonie ne posent pas de problème particulier. 
- Stades 7 à 5 : la transformation du sporozoïte directement en schizonte, sans division préalable en deux, n'est pas établie ; il s'agit cependant du mode d'évolution habituel des Coccidies.

- Schizogonies 5 et 7 : ces deux schizogonies observées en juillet diffèrent par la taille de leurs mérozoïtes; nous ignorons si elles se succèdent dans l'ordre que nous leur avons attribué.

- Schizogonies 9 à 15 : il est établi au cours de cette étude qu'il existe une schizogonie continue dans les glandes de Lieberkühn :

- schizontes de type A (9 à 13) présents tout au long des 24 h, mais plus nombreux au cours de la nuit que pendant la journée ;

- schizontes de type B (15) apparaissant un peu avant (novembre), ou en même temps (avril), que les schizontes de l'épithélium (17) et disparaissant avant eux.

- Schizogonie 17 : son apparition se fait un peu après celle de la schizogonie 15, en même temps que les premiers jeunes gamétocytes uninucléés et elle disparaît en même temps qu'eux. Nos observations montrent que les mérozoïtes issus de ces schizontes sont à l'origine des gamétocytes.

\section{3) Cycle prolongé à tropisme réticulo-endothélial}

a) Schéma du cycle ( $f \mathrm{~g} .3$ )

L'oocyste (41), lorsqu'il est ingéré par le Moineau, s'ouvre dans le tube digestif (1) ; le sporozoïte libéré (2) est pourvu de 2 cristalloïdes ; il pénètre dans une cellule du système réticulo-endothélial (3), où il subit une première division (4) en 2 éléments de taille égale avec expulsion d'un petit résidu nucléocytoplasmique. L'une des moitiés, pourvue d'un seul cristalloïde (5), pénètre dans une nouvelle cellule et commence une division schizogonique (6) ; celle-ci est de type ectomérogonique (7) : les futurs mérozoïtes bourgeonnent à la périphérie de la masse cytoplasmique, chaque bourgeon contenant un noyau et un petit cristalloïde ; certains de ces mérozoïtes (8) issus de la $1^{\text {re }}$ génération pénètrent dans une nouvelle cellule réticulo-endothéliale et se divisent par ectomérogonie (9) ; les mérozoïtes (10) issus de cette 2 e ectomérogonie sont dépourvus de cristallö̈de; ils pénètrent dans une cellule de l'épithélium des villosités intestinales pour se diviser par ectomérogonie (11) et libèrent des mérozoïtes (12), qui envahissent d'autres cellules de l'épithélium et donnent des schizontes de type $C$ (24). Les mérozoïtes libérés (25) pénètrent à leur tour dans une cellule de l'épithélium des villosités et se transforment en schizontes de type D (26).

Les mérozoites (27 et 31 ) sont à l'origine de la gamétogonie (28 à 34) ; le microgamétocyte (28) subit des divisions nucléaires (29) et à maturité libère des gamètes mâles ; le macrogamétocyte (32) augmente de taille (33) et, à maturité, est fécondé par un microgamète (34) ; l'oocyste ainsi formé (35) est libéré dans la lumière intestinale (36 à 38 ) et excrété dans le milieu extérieur.

Un certain nombre de mérozoïtes (13) issus de la première schizogonie prépatente (7) pénètrent dans une nouvelle cellule réticulo-endothéliale, qui est une cellule jeune, où ils se divisent par endomérogonie (14) ; plusieurs générations endoméro- 
goniques se succèdent (16-18) et aboutissent à la libération de mérozoïtes (19), qui pénètrent dans des petits lymphocytes et se transforment en petits trophozoites d'attente (20); ceux-ci se transforment progressivement en grands trophozoïtes d'attente (21) en même temps que leurs cellules hôtes paraissent se modifier en se dédifférenciant; un certain nombre de grands trophozoïtes d'attente sont activés tous les jours, se divisent (22) et libèrent des mérozoïtes (23), qui vont pénétrer dans une cellule de l'épithélium des villosités au début de la nuit ; ces mérozoïtes se divisent et donnent naissance à un schizonte de type C (24); la suite du cycle (25 à 38) est conforme à ce qui a été décrit plus haut (cf. cycle de type I. canaria).

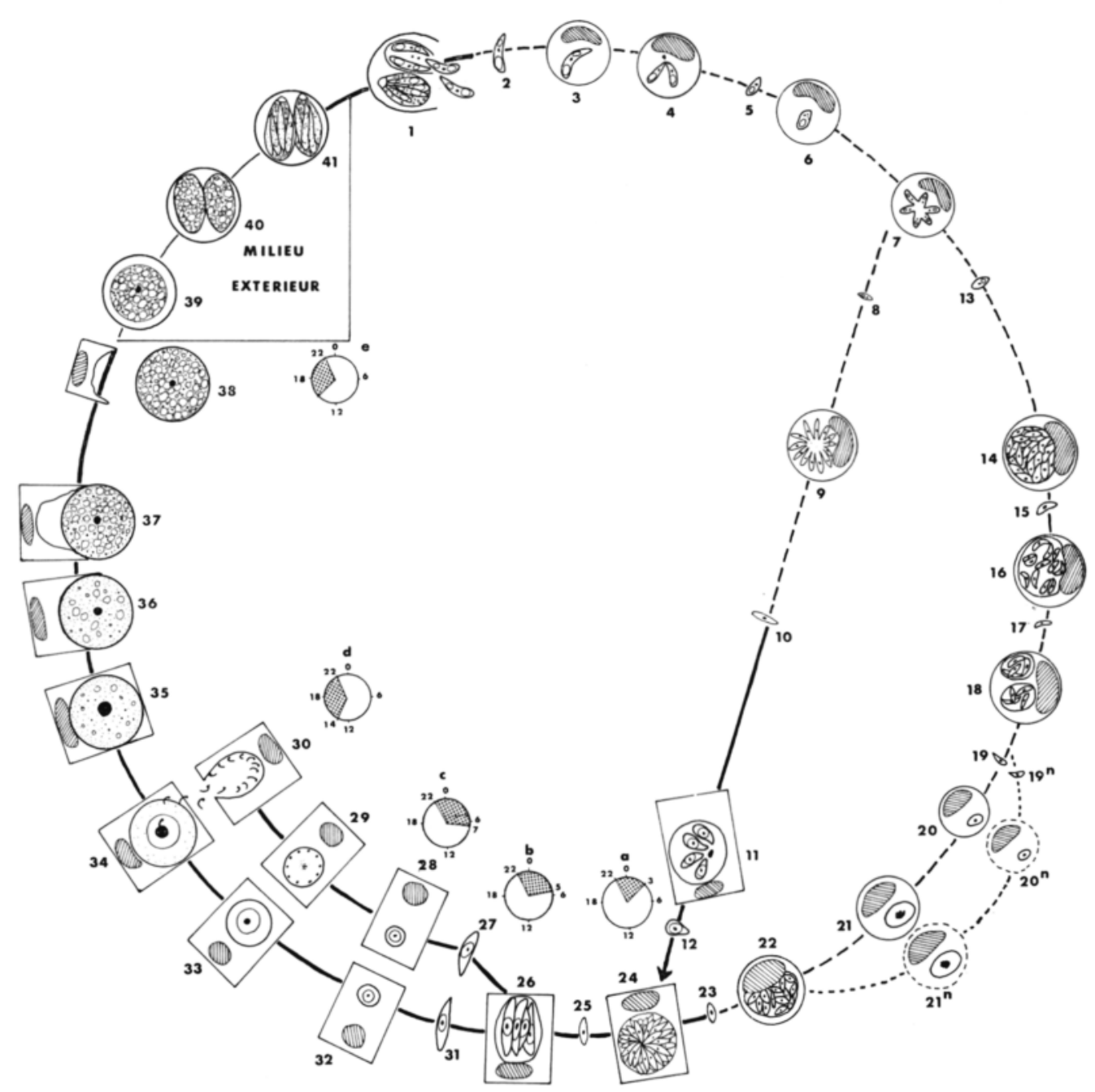

FIG. 3. - Cycle à tropisme réticulo-endothélial. 


\section{- Cycle nycthéméral (tableaux III et IV)}

Il varie de quelques heures selon la période de l'année ; en avril, les horaires sont les suivants :

- schizogonie 24 (type C) : de $22 \mathrm{~h}$ à $3 \mathrm{~h}$;

- schizogonie 26 (type D) : de $22 \mathrm{~h}$ à $5 \mathrm{~h}$;

- jeunes gamétocytes (28 et 32 ) : de $22 \mathrm{~h}$ à $7 \mathrm{~h}$;

- gamétocytes mûrs (30 et 34 ), oocystes intracellulaires (35) et oocystes dans la lumière de l'intestin (38) : de $14 \mathrm{~h}$ à $22 \mathrm{~h}$.

Les autopsies n'étant faites que toutes les deux heures, on ne peut s'étonner de voir deux stades supposés se succéder sur le schéma apparaître au même moment. Cela indique simplement que ces stades se succèdent rapidement.

- Durée d'évolution des phases du cycle

- période prépatente* : 9 jours ;

- cycle intestinal journalier (12 et 23 à 38 ) : 24 h;

- longévité des trophozoïtes d'attente : de 24 heures à 1 an ;

- période patente : infection chronique ;

- maturation des oocystes dans le milieu extérieur (39 à 41) : elle varie avec la température : $72 \mathrm{~h}$ à $23^{\circ} \mathrm{C}$.

b) Discussion

A l'exception du schizonte 11 , décrit par Box chez $I$. serini et que nous ne retrouvons pas chez le Moineau, tous les stades représentés dans le schéma sont observés par nous, dans les cultures (1 à 7), ou in vivo (2, 6, 9, 14 à 38).

- Rattachement des schizogonies intestinales 24 et 26 au cycle à tropisme réticuloendothélial

Les schizontes 24 , de type $\mathrm{C}$ et 26 , de type $\mathrm{D}$ sont rattachés à ce cycle pour les raisons exposées plus haut (cf. cycle à tropisme lieberkühnien).

\section{-. Succession des stades}

Ne sont traités ici que les stades prêtant à discussion, les phases classiques telles que la gamétogonie et la sporogonie ne posant pas de problème particulier.

\section{- Stades 1 à 7 :}

La division du sporozoïte en deux (4) et la formation du schizonte de lre génération ( 6 et 7) à partir de l'une des moitiés du sporozoïte, sont établies par Millet et coll. (1984) in vitro. Nous ignorons si l'un d'entre eux dégénère ou si l'évolution se poursuit après invasion d'une nouvelle cellule.

Les schizontes de première génération observés in vitro sont toujours isolés dans leurs cellules hôtes.

* Nous avons fait figurer pour la période prépatente les durées d'évolution signalées par Box, 1977, chez I. serini. 
- Schizogonies de la période prépatente ( 9 à 11 et 14 à 18) sont :

Pour Box, la période prépatente comprend 7 générations schizogoniques, qui

- dans le système réticulo-endothélial :

- $1^{\text {re }}$ génération ectomérogonique : $18 \mathrm{~h}$ à $2 \mathrm{j} 1 / 2$;

- $2^{\mathbf{e}}$ génération endomérogonique : $2 \mathrm{j} 1 / 2$;

- $3^{\mathrm{e}}$ génération endomérogonique : $3^{\mathrm{e}}-4^{\mathrm{e}} \mathrm{j}$;

- $4^{\mathrm{e}}$ génération ectomérogonique : $3^{\mathrm{e}} 6^{\mathrm{e}} \mathrm{j}$;

- $5^{\mathrm{e}}$ génération endomérogonique : $5^{\mathrm{e}}-8^{\mathrm{e}} \mathrm{j}$, qui donne naissance aux " single intramonocytic forms "(= trophozoites d'attente).

Les cinq générations se chevauchent, si bien que l'infection dans l'épithélium des villosités, qui succède à la $5^{\mathrm{e}}$ génération réticulo-endothéliale, débute dès le 8 jour.

- dans l'épithélium des villosités :

- 6e génération "endomérogonique » $^{\mathbf{e}}: 8^{\mathbf{e}} \mathbf{j}$;

- $7 \mathbf{e}$ génération ectomérogonique : $9 \mathrm{e} \mathbf{j}$.

Les travaux de Box montrent que la fin de la période prépatente est marquée par l'apparition simultanée d'une part de la $1^{\text {re }}$ gamétogonie, d'autre part des trophozoïtes d'attente.

Il nous paraît plus logique de considérer qu'il existe deux lignées, l'une ectomérogonique aboutissant directement à la première gamétogonie, l'autre endomérogonique à l'origine des trophozoïtes d'attente.

La chronologie fournie par Box est compatible avec cette interprétation :

- 1re génération ectomérogonique (schizonte 7 sur notre schéma) : 18 h à 2 j $1 / 2$;

$-2^{\mathrm{e}}$ génération ectomérogonique ( $4^{\mathrm{e}}$ génération de Box, schizonte 9 sur notre schéma) : $3^{\mathrm{e}}$ au $6^{\mathrm{e}} \mathbf{j}$;

- schizogonies intestinales ( $6^{\mathrm{e}}$ et $7^{\mathrm{e}}$ générations de Box, schizontes 11 et 24 sur notre schéma) : à partir du $8 \mathrm{e} \mathbf{j}$;

- schizogonies endomérogoniques $\left(2^{\mathrm{e}}, 3^{\mathrm{e}}\right.$ et $5^{\mathrm{e}}$ générations de Box, schizontes 14 à 18 sur notre schéma) : $2 \mathrm{j} 1 / 2$ à $8 \mathrm{j}$.

Cette évolution serait plus conforme à ce que l'on sait du cycle d'autres Coccidies où, en règle générale, l'endomérogonie est à l'origine de formes de latence et la division par ectomérogonie est à l'origine de la gamétogonie. Ainsi, par exemple :

- Hepatozoon domerguei Landau et coll., 1972, parasite de Reptiles :

- formation de kystes d'attente par endomérogonie;

- formation des gamétocytes à partir de schizontes ectomérogoniques.

* Nous interprétons d'une manière différente la nature de cette schizogonie : le schizonte figuré $f i g$. If et $I 7$ dans la description de Box, I977, nous paraît être ectomérogonique ; les mérozoïtes en apposition paraissent rayonner à partir du centre ; l'absence de corps résiduel notée par cet auteur est fréquente chez les schizontes ectomérogoniques mûrs. 
- Isospora felis Frenkel et Dubey, 1972 :

- formation de kystes d'attente par endomérogonie chez des Rongeurs ;

- formation des gamétocytes à partir de schizontes ectomérogoniques chez le Chat.

- Toxoplasma gondii:

- multiplications endomérogoniques rapides, suivies de formation de kystes d'attente chez différents hôtes (Mammifères, Oiseaux) ;

- multiplications endo- et ectomérogoniques rapides dans l'intestin du Chat (Dubey, Frenkel, 1972) ; comme chez le Moineau, ces deux types de multiplication se chevauchent : endomérogonie observée de la $12^{\mathrm{e}}$ heure au $15^{\mathrm{e}}$ jour ; ectomérogonie de la $28^{\mathrm{e}}$ heure au $15^{\mathrm{e}}$ jour, il n'y a, là encore, aucune preuve permettant d'affirmer qu'il y ait alternance d'ecto- et d'endomérogonies, et non coexistence de deux lignées parallèles.

\section{- Trophozoïtes d'attente 20 et 21 sur notre schéma}

L'interprétation selon laquelle les petits trophozoïtes d'attente grandissent et se transforment en grands trophozoïtes repose sur les observations suivantes : présence de stades intermédiaires entre les deux ; augmentation de la proportion des grands trophozoïtes en hiver en l'absence de toute schizogonie.

De plus, chez d'autres parasites, tels que Plasmodium cynomolgi (Krotoski et coll., 1980), parasite de Singe, on observe un phénomène comparable : la taille des " hypnozoïtes ", qui sont vraisemblablement des trophozoïtes en attente dans les hépatocytes de l'hôte, est plus faible au début de l'infestation que plusieurs semaines plus tard.

Un argument qui nous semble être décisif pour admettre l'homologie entre les trophozoïtes d'attente et les hypnozoïtes est l'action élective de la primaquine (cf. Grulet et coll., 1985 sous presse).

\section{- Schizogonie réticulo-endothéliale 22}

Dans le schéma proposé, la schizogonie intestinale nocturne a pour origine les grands trophozoïtes d'attente; nous admettons donc qu'un certain nombre d'entre eux évolue tous les jours et réensemence l'intestin. Le trophozoïte d'attente, qui est une forme immobile, doit donc se diviser (schizonte 22) pour donner naissance à des mérozoïtes mobiles capables d'envahir les cellules intestinales. Cette évolution serait conforme à ce qui a été observé chez $P$. cynomolgi, où l' "hypnozoïte " se transforme en schizonte, dont les mérozoïtes envahissent les globules rouges du sang, et déterminent une rechute de la parasitémie.

Nos observations ne permettent cependant pas de préciser le lieu et l'horaire de cette division : l'hypothèse la plus vraisemblable est qu'elle se produit à la fin de la journée dans l'axe conjonctif des villosités.

\section{- Schizogonies intestinales 24 et 26}

Comme nous l'avons vu dans la description du cycle, la schizogonie 24 (type C) est à l'origine de la schizogonie 26 (type D), elle-même à l'origine des gamétocytes. Les deux schizogonies sont observées en même temps au début de la nuit (tableau IV), 
mais la schizogonie 24 disparaît la première, alcrs que la schizogonie 26 persiste jusqu'à la formation des derniers gamétocytes. De plus, nos observations montrent que les mérozoïtes issus de schizontes de type D se transforment en gamétocytes.

\section{Conclusion}

L'unité des Sporozoaires est forte ; les mécanismes adaptatifs qui se sont mis en place pour assurer la pérennité de l'infection se retrouvent chez d'autres parasites :

- dans le Paludisme : nous avons rapproché les hypnozoïtes de Plasmodium vivax et $P$. cynomolgi des trophozoïtes d'attente des espèces à tropisme réticulo-endothélial. Cette homologie est confirmée par l'action élective de la primaquine (cf. Grulet et coll., 1985, sous presse) sur les trophozoites d'Isospora;

- dans la Toxoplasmose : le cycle évolutif de Toxoplasma gondii dans l'intestin du Chat, tel qu'il a été établi dans le travail de Dubey et Frenkel (1972) mérite à notre avis d'être vérifié et peut être remanié, car la biologie comparée des Sporozoaires plaide en faveur de deux lignées schizogoniques séparées : endomérogonique à l'origine des formes chroniques, et ectomérogonique à l'origine des formes sexuées.

\section{BIBLIOGRAPHIE}

Boughton D. C. : Diurnal gametic periodicity in avian Isospora. Am. J. Hyg., I933, I8, I6I.

Boughton D. C. : Studies on oocyst-production in avian coccidiosis - II. Chronic isosporan infections in the sparrow. Am.J.Hyg., I937,25, 203.

Box E. D. : Atoxoplasma associated with an Isospora oocyst in canaries. J. Protozool., I970, $r_{7}$, 39 I.

Box E. D. : Life cycle of two species in the Canary Serinus canarius Linnaeus. J. Protozool., I977, $24,57$.

Desser S. S. : An ultrastructural study of the asexual development of a presumed Isospora sp. in mononuclear, phagocytic cells of the evening grossbeak (Hesperiphona vespertina). J. Parasitol., 1980, 66, 601.

Dubey J. P., Frenkel J. K. : Cyst-Induced Toxoplasmosis in Cats. J. Protozool., I972, 19, I 55.

FAYER R. : Development of Sarcocystis fusiformis in the small intestine of dogs. J. Parasitol., 1974, 60,660 .

FRENKEL J. K., Dubey J. P. : Rodents as vectors for Feline Coccidia, Isospora felis and Isospora rivolta. $J$. Infect. Diseases, 1972, 125, 69.

Grulet O., Landau I., Baccam D. : Les Isospora du Moineau domestique; multiplicité des espèces. Ann. Parasitol. Hum. Comp., I982, 57, 209

Grulet O., Landau I., Millet P., Baccam D. : Les Isospora du Moineau : I. Compléments à l'étude systématique. Ann. Parasitol. Hum. Comp., 1986, 6r, I55-160.

Grulet O., Landau I., Millet P., Baccam D. : Les Isospora du Moineau : III. Action élective de la primaquine sur les formes d'attente. Ann. Parasitol. Hum. Comp., 1986, 61, 193-198.

HAMmoND D. M. : Life cycles and development of Coccidia, in D. M. Hammond and P. L. Long : The Coccidia, Eimeria, Isospora, Toxoplasma and Related-General, University Park Press, Baltimore, I973, $482 \mathrm{p}$.

Hutchison W. M., Dunachie J. F. : The life cycle of the coccidian parasite, Toxoplasma gondii, in the domestic cat. Trans. R. Soc. Trop. Med. Hyg., I97 I, 65, 380.

Jackson A. R. B. : The isolation of viable coccidial sporozoites. Parasitology, I964, 54, 87

Krotoski W. A., Krotoski D. M., Garnham P. C. C., Bray R. S., Killick-Kendrick R., Draper C. C., TARgetT G. A. T., Guy M. W. : Relapses in primate malaria : discovery of two populations of exoerythrocytic stages. Preliminary note. $\overline{B r}$. Med.J., I980, 23, I 53 .

Lambiotte M., Landau I., Thierry N., Miltgen F. : Développement de schizontes dans des hépatocytes de rat adulte en culture après infestation in vitro par des sporozoïtes de Plasmodium yoelii. C.R. Acad. Sci. Pavis, I981, 293, 43r. 
Landau I., Michel J. C., Chabaud A. G. : Cycle biologique d'Hepatozoon domerguei ; discussion sur les caractères fondamentaux d'un cycle de Coccidie. $Z$. Parasitenk., 1972, 38, 250.

LANDAU I. : Diversité des mécanismes assurant la pérennité de l'infection chez les Sporozoaires Coccidiomorphes. Mém. Mus. natn. Hist. nat., nouv. sér., Sér. A. Zool., 1973, 77, 62.

Landau I., Marteau M., Golvan Y., Chabaud A. G., Boulard Y. : Hétéroxénie chez les Coccidies intestinales de Poissons. C.R. Acad. Sci. Paris., I975, 281 , Sér. D, I 72 I

LANDAU I. : Hypothèses sur la phylogénie des cycles et spectres d'hôtes des Coccidiomorphes. In : Deuxième Symposium sur la Spécificité parasitaire des parasites de Vertébrés. Mém. Mus. natn. Hist. nat., nouv. sér., Sér. A. Zool., I982, I $23,326$.

Millet P., Baxter J., Landau I. : Division initiale de sporozoïtes d'une $I_{\text {sospora de Moineau en }}$ culture cellulaire. Ann. Parasitol. Hum. Comp., I984, 59, 321 .

Solangi A. M., Overstreet R. M. : Biology and pathogenesis of the Coccidium Eimeria funduli infecting killifishes. J. Parasitol., I980, 66, 51 3 .

Stabler R., Kitzmiller N. J. : Isospora petrochelidon sp. n. (Protozoa : Eimeriidac) from the cliff swallow, Petrochelidon pyrrhonota. J. Protozool., 1972, 19, 249. 